\title{
A fractional model in exploring the role of fear in mass mortality of pelicans in the Salton Sea
}

\author{
Ankur Jyoti Kashyap* Debasish Bhattacharjee, and Hemanta Kumar Sarmah \\ Department of Mathematics, Gauhati University, Assam, India \\ ajkashyap.maths@gmail.com,debabh2@gmail.com, hsarmah[at]gauhati[dot]ac[dot]in
}

\section{ARTICLE INFO}

\section{Article History:}

Received 9 June 2021

Accepted 3 December 2021

Available 31 December 2021

\section{Keywords:}

Epidemiology

Fractional-order system

Fear effect

Stability

Hopf bifurcation

Numerical simulation

AMS Classification 2010:

37M10; 37N25; 37G15; 34K37; $34 \mathrm{~K} 99$

\section{ABSTRACT}

The fear response is an important anti-predator adaptation that can significantly reduce prey's reproduction by inducing many physiological and psychological changes in the prey. Recent studies in behavioral sciences reveal this fact. Other than terrestrial vertebrates, aquatic vertebrates also exhibit fear responses. Many mathematical studies have been done on the mass mortality of pelican birds in the Salton Sea in Southern California and New Mexico in recent years. Still, no one has investigated the scenario incorporating the fear effect. This work investigates how the mass mortality of pelican birds (predator) gets influenced by the fear response in tilapia fish (prey). For novelty, we investigate a modified fractional-order eco-epidemiological model by incorporating fear response in the prey population in the Caputo-fractional derivative sense. The fundamental mathematical requisites like existence, uniqueness, non-negativity and boundedness of the system's solutions are analyzed. Local and global asymptotic stability of the system at all the possible steady states are investigated. Routh-Hurwitz criterion is used to analyze the local stability of the endemic equilibrium. Fractional Lyapunov functions are constructed to determine the global asymptotic stability of the disease-free and endemic equilibrium. Finally, numerical simulations are conducted with the help of some biologically plausible parameter values to compare the theoretical findings. The order $\alpha$ of the fractional derivative is determined using Matignon's theorem, above which the system loses its stability via a Hopf bifurcation. It is observed that an increase in the fear coefficient above a threshold value destabilizes the system. The mortality rate of the infected prey population has a stabilization effect on the system dynamics that helps in the coexistence of all the populations. Moreover, it can be concluded that the fractional-order may help to control the coexistence of all the populations.

\section{Introduction}

The conventional notion that predator affects the prey population only through direct killing has been changed to a great extent in recent past [1]. The population dynamics of the prey is more affected by indirect interaction with a predator as compared to direct killing [2]. In addition to killing, predators often elicit a fear response in the prey population which brings about many psychological and physiological changes in the prey [3].
The primary line of anti-predator behaviour is to avoid detection. Due to predation risk, prey may compromise with the source and choice of foraging, which ultimately affect personal or community growth and thereby affecting reproduction [2, 4]. Fear may affect the physiological condition of the juvenile prey, and this could leave a negative effect on their survival as adults 4,.5]. In the experiment conducted by Zanette et al. 6 , it is observed that song sparrows had a reduced 
growth rate due to the perceived predation risk even after the absence of direct killing. So, predators frequently affect the prey, indirectly, enforcing a stressful life.

Similarly, fear-induced phenomena can also be found in different fishes in marine ecology. In rainbow trout species (Oncorhynchus mykiss), for example, reproduction timing may vary due to stress like disturbance or handling. However, Tilapia (Oreochromis niloticus), when subjected to a stressed environment, show different types of the psychology of reproduction depending on its maturity [7]. It is observed that the minimum threshold of stimulus that is required to elicit a behavioral response in prey is lower for fishes (prey individuals) who are previously exposed to higher levels of predation risk. Fish actually optimizes their feeding rate under the constraints of predation severity leading to mortality [8].

Physiological changes are observed in the body of the prey as a response to the actual presence or background knowledge of predators. These physiological changes are brought about by hormonal or neuronal changes that bring about different responses in organ systems and ultimately lead to altered reproductive capacity. Showing antipredator behaviour costs a rebalance in energy allocation and subsequently could affect the reproduction process [9].

Salton sea is a very stressed environment for fish. From 1970 onwards, the total fish biomass of Salton sea has been crashed many times due to three physiological stressors viz. extreme temperature fluctuation, increasing salinity, and high sulphide levels and anoxia associated with mixing events [10]. This stress environment affects the vital life functions of fish, mainly population growth via reproduction [9]. Although the role of stressors directly from the environment has an active role in the life cycle of fish species at Salton sea, another stress, that is, fear of predation, can not be ruled out considering recent discussions in literature [2].

Avian botulism(Clostridium botulinum type C) is a regular outbreak causing sizeable mortality among the piscivorous birds of the Salton sea since the twentieth century [11]. In 1996, around 9000 white Pelicans and around 1200 brown Pelicans were killed due to this dreaded disease. However, the mortality number has dropped significantly, and white pelicans were affected less in mortality than brown pelicans $[12]$. Type $\mathrm{C}$ botulinum toxin formed inside the gastrointestinal tracts of Tilapia, infected by a variety of bacteria like vibrato, is considered to be the main cause of death among pelicans.
Mathematical modeling has a very important role in studying the interaction among the predator and prey species. After the pioneering work of Kermack and Mc Kendrick on SIRS type, epidemiological modeling has been studied widely in recent years by various researchers $[13$ 17]. Mathematical modeling of the fear effect in prey species was first proposed by Wang et al. [18] in the year 2016. Subsequently, some fear-induced mathematical studies of predator-prey interaction have been carried out [19 21]. In their study, Hossain et al. investigated the effect of fear in a threespecies intraguild predation model. Their analysis revealed that fear could stabilize the chaos produced due to omnivory predators [19]. Predators that follow cooperation strategies while hunting also creates fear upon the prey. Combining hunting cooperation (by predators) and fear effect (in prey), Pal et al. investigated a Lotka-Volterra type predator-prey model. Their study shows that an increase in the hunting cooperation induced fear may destabilize the system and produce periodic solutions via a Hopf-bifurcation 20. Panday et al. in 21 studied the impact of fear in a tri-trophic food chain model. They observe that fear can stabilize the system from chaos to stable focus through the period halving phenomenon. Till now, we have not come across any literature where the role of fear has been analyzed in the case of the marine ecosystem. This has motivated us for the present investigation.

In recent years, researchers have shown more interest in using fractional-order differential equations (FDE) in mathematical modeling rather than integro-differential equations (IDE). FDEs can be used to model universal phenomena with greater precision 23, 24]. In [23, Heymans et al., through a series of examples, have demonstrated that it is possible to attribute physical meaning to initial conditions expressed in terms of Riemann-Liouville fractional derivatives. Unlike IDEs, FDEs are non-local operators where the succeeding state of any function depends not only on their existing state but also on all preceding states 25 26]. In addition, classical IDEs are incapable of providing data between two distinct integer values. To overcome such restrictions, various types of fractional-order operators were adopted in the available literature [27,28]. Moustafa et al. in 29] investigated an eco-epidemiological model with disease in the prey species in terms of Caputo fractional derivative. Khan et al. in [26] investigated a fractional SIR model with a generalized incidence rate using both Caputo and the recently 
developed Atangana-Baleanu-Caputo (ABC) derivative [28]. The $\mathrm{ABC}$ derivative having nonsingular and non-local kernel contains a strong memory effect of the system. Recently, Singh et al. investigated a fractional guava fruit model involving $\mathrm{ABC}$ derivative for investigating the interaction between guava pests and natural enemies [30]. Tuan et al. studied the existence and uniqueness of a mild solution to an initial value problem for a fractional Rayleigh-Stokes equation driven by fractional Brownian motion [31]. Fuzzy $\mathrm{ABC}$ fractional derivative, fuzzy $\mathrm{ABC}$ fractional initial value problems, and fuzzy $\mathrm{ABC}$ solutions are discussed and utilized for the first time in 32$]$. Bonyah et al. studied a fractional optimal control model of coronavirus in ABC derivative sense [33].

Mathematical research work on large-scale mortality of pelicans in the Salton Sea was first carried out by Bairagi et al. [34] in 2001. Throughout their article, the authors presumed that pelicans only come into contact with infected tilapia. Using the same perspective, Greenhalgh et al. [36] in 2007 proposed a ratio-dependent predatorprey interaction model ignoring the predation of susceptible prey. In their research work, they adopted a purely logistic growth function of the susceptible prey. The predators do not have any alternative food resources, and they prey only on infected prey. So, their carrying capacity depends only on infected prey. In their research, Chattopadhyay et al. [35] modified the previous study by introducing an interaction between pelicans and susceptible fish. The authors presumed that the death rate of the pelicans is increased due to feeding on infected fish. Later in 2017, Greenhalgh et al. 37] modified their earlier studies in [36] by taking into account that predators feed on both the susceptible and infected preys(tilapia). Furthermore, they presumed that the diseased prey significantly influences the growth rate of susceptible prey and the carrying capacity of the predator is dependent on the total number of prey (tilapia). To make the discussions more realistic and novel, we have considered the fear effect in the prey (tilapia) due to predation (by pelicans), since Tilapia (Oreochromis niloticus) under stressful circumstances (like predation risk) react by boosting or completely hindering reproduction [7].

In this paper, we extend the mathematical model proposed by Greenhalgh in [37] by incorporating the fear effect in the susceptible prey (tilapia) in terms of Caputo fractional derivative. The integer-order derivative does not contain the complete memory, and it does not describe the physical behavior of the model. The memory effect in FDEs that provides data between two distinct integer values motivates us to study the model using a fractional derivative. Besides terrestrial ecosystems, the fear effect also influences marine ecosystems. Therefore we are interested in exploring the complex dynamics of the critical ecosystem of the Salton Sea (which became a dangerous habitat for birds during the 1990s) with fear effect. Additionally, the fear effect induced in the prey population due to predation risk makes the scenario a novel one and biologically more realistic and meaningful. The paper is organized as follows:

In section 2, we describe a modified predator-prey interaction model with fear effect involving Caputo fraction derivatives. In section 3 , we provide some mathematical preliminaries used for analytical discussions of our model. Fundamental mathematical results like the existence, uniqueness, non-negativity and boundedness solutions of the modified model are carried out throughout section 4 . In sections 5 and 6 , the modified model's equilibrium points and their local stability are analyzed. In sections 7 and 8, the global stability of the disease-free equilibrium, positive equilibrium, and condition for Hopf bifurcation at the diseasefree equilibrium is discussed. In section 9, numerical simulations are carried out using biologically feasible parameters. Finally, in section 10, a summary of the outcomes obtained from the current study is provided. The conclusions derived are purely on the basis of theoretical results. Experimental verification will suggest modification required in fundamental assumptions.

\section{Model formulation}

In this section, we discuss a modified form of a predator-prey interaction model initially forwarded by Greenhalgh et al. 37]. The model in 37] is based on the critical ecosystem of the Salton Sea located in Southern California, New Mexico, where pelicans and tilapia are the predator and prey, respectively. The disease is assumed to spread among the prey through close contact. The vibrio-infected prey is classified into susceptible and infected prey and are represented by $S(T)$ and $I(T)$ respectively. So, at any instant $T$, the total number of prey (tilapia) population is $N(T)=S(T)+I(T)$. Only susceptible prey (tilapia) take part in reproducing offsprings, and population growth is in logistic fashion with a carrying capacity of $k>0$. The predators (pelicans) feed on both susceptible and infected prey, preferably the infected ones, since they are easily catchable. They assumed that the predators feed 
on the prey with a ratio-dependent functional response. With these basic assumptions, the model is,

$$
\begin{aligned}
& \frac{d S}{d T}=r S\left(1-\frac{S+I}{k}\right)-\lambda S I-\frac{p Y S}{m Y+S} \\
& \frac{d I}{d T}=\lambda S I-\frac{c Y I}{m Y+I}-\gamma I \\
& \frac{d Y}{d T}=\delta Y\left(1-\frac{h Y}{S+I}\right)
\end{aligned}
$$

with the initial condition $S(0) \geq 0, I(0) \geq$ $0, Y(0) \geq 0$ where,

$r$ : rate of growth of the prey species in the reproducing population group,

$k$ : total capacity of the system,

$\lambda$ : the disease transmission coefficient, $p, c$ : catching rate of predators (pelicans) towards susceptible and infected prey (tilapia), respectively,

$m$ : a strictly positive constant,

$\gamma$ : the mortality rate of infected prey per capita,

$\delta$ : rate of growth of the predator (pelican) species per capita,

$h$ : a constant which is related to the densitydependent death rate of the predator(pelican) population.

The modified fractional-order model of the system (1) is presented as follows

$$
\begin{aligned}
& { }^{c} D_{t}^{\alpha} S(T)=r S\left(1-\frac{S+I}{k}\right)-\lambda S I-\frac{p Y S}{m Y+S} \\
& { }^{c} D_{t}^{\alpha} I(T)=\lambda S I-\frac{c Y I}{m Y+I}-\gamma I \\
& { }^{c} D_{t}^{\alpha} Y(T)=\delta Y\left(1-\frac{h Y}{S+I}\right)
\end{aligned}
$$

Since the induced fear in the prey (tilapia) due to predation risk reduces their reproduction rate, therefore we modify the first equation of system (2) by multiplying the breeding rate $r$ with a factor $g(f, Y)$ as below,

$$
\begin{aligned}
{ }^{c} D_{t}^{\alpha} S(T) & =r S\left(1-\frac{S+I}{k}\right) g(f, Y)-\lambda S I \\
& -\frac{p Y S}{m Y+S}
\end{aligned}
$$

where $Y$ describes the biomass of the predator (pelican) and $f$ describes the strength of fear due to predation risk in the prey (tilapia). To make $f, Y$ and $g(f, Y)$ biologically feasible it is appropriate to assume that 18

$$
\begin{array}{r}
g(0, Y)=1, g(f, 0)=1, \lim _{f \rightarrow \infty} g(f, Y)=0, \\
\lim _{Y \rightarrow \infty} g(f, Y)=0, \frac{\partial g(f, Y)}{\partial f}<0, \frac{\partial g(f, Y)}{\partial Y}<0 .
\end{array}
$$

Here we consider $g(f, Y)=\frac{1}{1+f Y}$ which satisfies condition (4). Then the system (2) becomes:

$$
\begin{aligned}
{ }^{c} D_{t}^{\alpha} S(T) & =r S\left(1-\frac{S+I}{k}\right)\left(\frac{1}{1+f Y}\right)-\lambda S I \\
& -\frac{p Y S}{m Y+S} \\
{ }^{c} D_{t}^{\alpha} I(T) & =\lambda S I-\frac{c Y I}{m Y+I}-\gamma I \\
{ }^{c} D_{t}^{\alpha} Y(T) & =\delta Y\left(1-\frac{h Y}{S+I}\right)
\end{aligned}
$$

with the initial condition $S(0) \geq 0, I(0) \geq$ $0, Y(0) \geq 0$.

Now define $T=\lambda t, r_{1}=\frac{r}{\lambda}, p_{1}=\frac{p}{\lambda}, \gamma_{1}=\frac{\gamma}{\lambda}, \delta_{1}=$ $\frac{\delta}{\lambda}, c_{1}=\frac{c}{\lambda}$. Then the system (5) reduces to

$$
\begin{aligned}
{ }^{c} D_{t}^{\alpha} S(t) & =r_{1} S\left(1-\frac{S+I}{k}\right)\left(\frac{1}{1+f Y}\right)-S I \\
& -\frac{p_{1} Y S}{m Y+S} \\
{ }^{c} D_{t}^{\alpha} I(t) & =S I-\frac{c_{1} Y I}{m Y+I}-\gamma_{1} I \\
{ }^{c} D_{t}^{\alpha} Y(t) & =\delta_{1} Y\left(1-\frac{h Y}{S+I}\right)
\end{aligned}
$$

with the initial condition $S(0) \geq 0, I(0) \geq$ $0, Y(0) \geq 0$.

\section{Mathematical preliminaries}

Throughout this section, we present a few preliminary definitions as well as some important lemmas for Caputo fractional derivative $22,24,38,39$.

Definition 1. [24] Let $g$ be any function such that $g \in \mathcal{C}^{n}\left(\left[t_{0},+\infty\right), \mathbb{R}\right)$ then the Caputo fractional derivative of $g$ having order $\alpha$ is defined by

$$
{ }_{t_{0}}^{c} D_{t}^{\alpha} g(t)=\frac{1}{\Gamma(n-\alpha)} \int_{t_{0}}^{t} \frac{g^{(n)}(s)}{(t-s)^{\alpha-n+1}} d s
$$

where $\Gamma($.$) is the Gamma function, n$ is a nonnegative integer such that $n-1<\alpha<n$ and $t \geq t_{0}$. In particular, when $0<\alpha<1$

$$
{ }_{t_{0}}^{c} D_{t}^{\alpha} g(t)=\frac{1}{\Gamma(1-\alpha)} \int_{t_{0}}^{t} \frac{g^{\prime}(s)}{(t-s)^{\alpha}} d s
$$


Lemma 1. $[38] \operatorname{Let} g(t) \in \mathcal{C}[a, b]$ and ${ }_{t_{0}}^{c} D_{t}^{\alpha} g(t) \in$ $\mathcal{C}[a, b], 0<\alpha \leq 1$, then

(i) for each $t \in[a, b], g(t)$ is non-decreasing provided ${ }_{t_{0}}^{c} D_{t}^{\alpha} g(t) \geq 0, a<t<b$.

(ii) for each $t \in[a, b], g(t)$ is non-increasing provided ${ }_{t_{0}}^{c} D_{t}^{\alpha} g(t) \leq 0, a<t<b$.

Lemma 2. 24] Consider the Cauchy problem

$$
\begin{aligned}
{ }_{a}^{c} D_{t}^{\alpha} \hat{x}(t) & =\lambda \hat{x}(t)+g(t) \\
\hat{x}(a) & =b(b \in \mathbb{R})
\end{aligned}
$$

with $0<\alpha<1$ and $\bar{\lambda} \in \mathbb{R}$. Then the solution is of the form

$$
\begin{aligned}
\hat{x}(t) & =b E_{\alpha}\left[\bar{\lambda}(t-a)^{\alpha}\right] \\
& +\int_{a}^{t}(t-s)^{(\alpha-1)} E_{\alpha, \alpha}\left[\bar{\lambda}(t-s)^{\alpha}\right] g(s) d s
\end{aligned}
$$

while the solution to the problem

$$
\begin{aligned}
{ }_{a}^{c} D_{t}^{\alpha} \hat{x}(t) & =\bar{\lambda} \hat{x}(t) \\
\hat{x}(a) & =b(b \in \mathbb{R})
\end{aligned}
$$

is given by

$$
\hat{x}(t)=b E_{\alpha}\left[\bar{\lambda}(t-a)^{\alpha}\right]
$$

The preceding lemma is quite important to verify that the system (6) is uniformly bound which is the generalization of the Lemma 2 provided in 40 .

Lemma 3. [22] Consider a function $\bar{u}(t)$ continuous on $\left[t_{0},+\infty\right)$ satisfying

$$
\begin{gathered}
{ }_{t_{0}}^{c} D^{\alpha} \bar{u}(t) \leq-\bar{\lambda} \bar{u}(t)+\mu \\
\bar{u}\left(t_{0}\right)=\bar{u}_{t_{0}}
\end{gathered}
$$

where $0<\alpha<1,(\bar{\lambda}, \mu) \in \mathbb{R}^{2}$, and $\bar{\lambda} \neq 0$ and $t_{0} \geq 0$ is the initial time. Then

$$
\bar{u}(t) \leq\left(\bar{u}_{t_{0}}-\frac{\mu}{\bar{\lambda}}\right) E_{\alpha}\left[-\bar{\lambda}\left(t-t_{0}\right)^{\alpha}\right]+\frac{\mu}{\bar{\lambda}}
$$

Lemma 4. Consider a function $\bar{u}(t)$ continuous on $\left[t_{0},+\infty\right)$ satisfying

$$
\begin{gathered}
{ }_{t_{0}}^{c} D_{t}^{\alpha} \bar{u}(t) \geq \bar{\lambda} \bar{u}(t)-\mu \\
\bar{u}\left(t_{0}\right)=\bar{u}_{t_{0}}
\end{gathered}
$$

where $0<\alpha<1,(\bar{\lambda}, \mu) \in \mathbb{R}^{2}$, and $\bar{\lambda} \neq 0$ and $t_{0} \geq 0$ is the initial time. Then

$$
\bar{u}(t) \geq\left(\bar{u}_{t_{0}}-\frac{\mu}{\bar{\lambda}}\right) E_{\alpha}\left[\bar{\lambda}\left(t-t_{0}\right)^{\alpha}\right]+\frac{\mu}{\bar{\lambda}}
$$

Proof. This lemma can be proved using the similar approach used in the proof of the lemma (3).
Lemma 5. [39] Consider $\hat{x}(t) \in \mathbb{R}_{+}$be a continuous and derivable function. Then for any $t \geq t_{0}$

$$
\begin{aligned}
& { }_{t_{0}}^{c} D_{t}^{\alpha}\left[\hat{x}(t)-\hat{x}^{*}-\hat{x}^{*} \ln \frac{\hat{x}(t)}{\hat{x}^{*}}\right] \\
& \leq\left(1-\frac{\hat{x}^{*}}{\hat{x}(t)}\right){ }_{t_{0}}^{c} D_{t}^{\alpha} \hat{x}(t), \\
& \hat{x}^{*} \in \mathbf{R}_{+}, \forall \alpha \in(0,1)
\end{aligned}
$$

\section{Mathematical analysis}

In this section, we present the fundamental mathematical requisites like the existence, uniqueness, non-negativity, and boundedness of the solutions, as desired in any population dynamics.

\subsection{Existence and Uniqueness of the system}

We investigate the existence and uniqueness of the solutions of the fractional-order system $(6)$ in the region $\mathcal{B} \times\left[t_{0}, T\right]$ where

$$
\begin{gathered}
\mathcal{B}=\left\{(S, I, Y) \in \mathbb{R}^{3}: \max \{|S|,|I|,|Y|\} \leq \Psi,\right. \\
\left.\quad \min \{|S|,|I|,|Y|\} \geq \Psi_{0}\right\} \text { and } T<+\infty .
\end{gathered}
$$

Theorem 1. For each $X_{0}=\left(S_{0}, I_{0}, Y_{0}\right) \in \mathcal{B}$, there exists a unique solution $X(t) \in \mathcal{B}$ of the fractional-order system (6) with initial condition $X_{0}$, which is defined for all $t \geq 0$

Proof. We denote $X=(S, I, Y)$ and $\bar{X}=$ $(\bar{S}, \bar{I}, \bar{Y})$.

Consider a mapping

$M(X)=\left(M_{1}(X), M_{2}(X), M_{3}(X)\right)$ and

$$
\begin{aligned}
M_{1}(X) & =r_{1} S\left(1-\frac{S+I}{k}\right)\left(\frac{1}{1+f Y}\right)-S I \\
& -\frac{p_{1} Y S}{m Y+S} \\
M_{2}(X) & =S I-\frac{c_{1} Y I}{m Y+I}-\gamma_{1} I \\
M_{3}(X) & =\delta_{1} Y\left(1-\frac{h Y}{S+I}\right)
\end{aligned}
$$

For any $X, \bar{X} \in \mathcal{B}$ it follows from equation (14) that

$$
\begin{aligned}
& \|M(X)-M(\bar{X})\| \\
& =\left|M_{1}(X)-M_{1}(\bar{X})\right| \\
& +\left|M_{2}(X)-M_{2}(\bar{X})\right|+\left|M_{3}(X)-M_{3}(\bar{X})\right|
\end{aligned}
$$




$$
\begin{aligned}
& \left|M_{1}(X)-M_{1}(\bar{X})\right| \\
& =\mid r_{1} S\left(1-\frac{S+I}{k}\right)-S I-\frac{p_{1} Y S}{m Y+S}-r_{1} \bar{S}\left(1-\frac{\bar{S}+\bar{I}}{k}\right) \\
& +\bar{S} \bar{I}+\frac{p_{1} \bar{Y} \bar{S}}{m \bar{Y}+\bar{S}} \mid \\
& =\left|r_{1}(S-\bar{S})-\frac{r_{1}}{k} S(S+I)-S I-\frac{p_{1} Y S}{m Y+S}-\frac{r_{1}}{k} \bar{S}(\bar{S}+\bar{I})+\bar{S} \bar{I}+\frac{p_{1} \bar{Y} \bar{S}}{m \bar{Y}+\bar{S}}\right| \\
& \leq\left|r_{1}(S-\bar{S})\right|+\frac{r_{1}}{k}\left|\left(S^{2}-\bar{S}^{2}\right)\right|+\left(\frac{r_{1}}{k}+1\right)|(S I-\bar{S} \bar{I})|+\frac{p_{1}}{m}|(S-\bar{S})| \\
& +p_{1}|(Y-\bar{Y})| \\
& \leq r_{1}|S-\bar{S}|+\frac{2 r_{1}}{k} \Psi|S-\bar{S}|+\left(\frac{r_{1}}{k}+1\right) \Psi|S-\bar{S}|+\left(\frac{r_{1}}{k}+1\right) \Psi|I-\bar{I}| \\
& +\frac{p_{1}}{m}|S-\bar{S}|+p_{1}|Y-\bar{Y}| \\
& \left|M_{2}(X)-M_{2}(\bar{X})\right| \\
& =\left|(S I-\bar{S} \bar{I})-\gamma_{1}(I-\bar{I})-\left(\frac{c_{1} Y I}{m Y+I}\right)+\left(\frac{c_{1} \bar{Y} \bar{I}}{m \bar{Y}+\bar{I}}\right)\right| \\
& \leq \Psi|S-\bar{S}|+\Psi|I-\bar{I}|+\gamma_{1}|I-\bar{I}|+\frac{c_{1}}{m}|I-\bar{I}|+c_{1}|Y-\bar{Y}| \\
& \left|M_{3}(X)-M_{3}(\bar{X})\right|=\left|\delta_{1} Y\left(1-\frac{h Y}{S+I}\right)-\delta_{1} \bar{Y}\left(1-\frac{h \bar{Y}}{\bar{S}+\bar{I}}\right)\right| \\
& \leq \delta_{1}|Y-\bar{Y}|+\delta_{1} h\left|\frac{Y^{2}}{S+I}-\frac{\bar{Y}^{2}}{\bar{S}+\bar{I}}\right| \\
& \leq \delta_{1}|Y-\bar{Y}|+\delta_{1} h\left|\frac{\left(Y^{2}-\bar{Y}^{2}\right)(S+I)-Y^{2}(S-\bar{S})-Y^{2}(I-\bar{I})}{(S+I)(\bar{S}+\bar{I})}\right| \\
& \leq \delta_{1}|Y-\bar{Y}|+\delta_{1} h\left|\frac{\left(Y^{2}-\bar{Y}^{2}\right)}{(\bar{S}+\bar{I})}\right|+\delta_{1} h\left|\frac{Y^{2}(S-\bar{S})}{(S+I)(\bar{S}+\bar{I})}\right|+\delta_{1} h\left|\frac{Y^{2}(I-\bar{I})}{(S+I)(\bar{S}+\bar{I})}\right| \\
& \leq \delta_{1}|Y-\bar{Y}|+\frac{\delta_{1} h \Psi}{\Psi_{0}}|Y-\bar{Y}|+\frac{\delta_{1} h}{4 \Psi_{0}^{2}}\left|Y^{2}(S-\bar{S})\right|+\frac{\delta_{1} h}{4 \Psi_{0}^{2}}\left|Y^{2}(I-\bar{I})\right| \\
& \leq \delta_{1}|Y-\bar{Y}|+\frac{\delta_{1} h \Psi}{\Psi_{0}}|Y-\bar{Y}|+\frac{\delta_{1} h \Psi^{2}}{4 \Psi_{0}^{2}}|(S-\bar{S})|+\frac{\delta_{1} h \Psi^{2}}{4 \Psi_{0}^{2}}\left|Y^{2}(I-\bar{I})\right|
\end{aligned}
$$

Then equation (15) becomes,

$$
\begin{aligned}
\|M(X)-M(\bar{X})\| & \leq r_{1}|S-\bar{S}|+\frac{2 r_{1}}{k} \Psi|S-\bar{S}|+\left(\frac{r_{1}}{k}+1\right) \Psi|S-\bar{S}|+\left(\frac{r_{1}}{k}+1\right) \Psi|I-\bar{I}| \\
& +\frac{p_{1}}{m}|S-\bar{S}|+p_{1}|Y-\bar{Y}|+\Psi|S-\bar{S}|+\Psi|I-\bar{I}|+\gamma_{1}|I-\bar{I}| \\
& +\frac{c_{1}}{m}|I-\bar{I}|+c_{1}|Y-\bar{Y}|+\delta_{1}|Y-\bar{Y}|+2 \delta_{1} h \Psi|Y-\bar{Y}|+\delta_{1}|Y-\bar{Y}| \\
& +\frac{\delta_{1} h \Psi}{\Psi_{0}}|Y-\bar{Y}|+\frac{\delta_{1} h \Psi^{2}}{4 \Psi_{0}^{2}}|(S-\bar{S})|+\frac{\delta_{1} h \Psi^{2}}{4 \Psi_{0}^{2}}\left|Y^{2}(I-\bar{I})\right| \\
& \leq\left\{r_{1}+\left(\frac{3 r_{1}}{k}+2\right) \Psi+\frac{p_{1}}{m}+\frac{\delta_{1} h \Psi^{2}}{4 \Psi_{0}^{2}}\right\}|S-\bar{S}|+\left\{\left(\frac{r_{1}}{k}+2\right) \Psi+\gamma_{1}+\frac{c_{1}}{m}\right. \\
& \left.+\frac{\delta_{1} h \Psi^{2}}{\Psi_{0}^{2}}\right\}|I-\bar{I}|+\left\{p_{1}+c_{1}+\delta_{1}+\frac{\delta_{1} h \Psi}{\Psi_{0}}\right\}|Y-\bar{Y}| \\
\|M(X)-M(\bar{X})\| & \leq L\|X-\bar{X}\|
\end{aligned}
$$


where

$$
\begin{aligned}
& L=\max \left\{\left(r_{1}+\left(\frac{3 r_{1}}{k}+2\right) \Psi+\frac{p_{1}}{m}+\frac{\delta_{1} h \Psi^{2}}{4 \Psi_{0}^{2}}\right),\right. \\
& \quad\left(\frac{r_{1}}{k}+2\right) \Psi+\gamma_{1}+\frac{c_{1}}{m}+\frac{\delta_{1} h \Psi^{2}}{\Psi_{0}^{2}}, \\
& \left.p_{1}+c_{1}+\delta_{1}+\frac{\delta_{1} h \Psi}{\Psi_{0}}\right\}
\end{aligned}
$$

Therefore $M(X)$ obeys Lipschitz condition which implies the existence and uniqueness of solution of the fractional-order system (6).

\subsection{Non-negativity and boundedness}

Consider the set

$$
\begin{aligned}
\mathcal{B}_{+} & =\left\{(S, I, Y) \in \mathcal{B}: S \in \mathbb{R}_{+}, I \in \mathbb{R}_{+}\right. \\
& \text {and } \left.Y \in \mathbb{R}_{+}\right\}
\end{aligned}
$$

where $\mathbb{R}_{+}$is the set of all non-negative real numbers.

Theorem 2. All the solutions of the fractionalorder system (6) initiating in the region $\mathcal{B}_{+}$are non-negative and bounded uniformly.

Proof. For the proof we follow the approach used in $[22$.

First, we prove that the solutions $S(t)$ that initiate in $\mathcal{B}_{+}$are non-negative i.e., $S(t) \geq 0$ for all $t \geq t_{0}$. Let us assume that is not true, then there exists $t>t_{0}$ such that

$$
\begin{aligned}
S(t) & >0, t_{0} \leq t<t_{1} \\
S\left(t_{1}\right) & =0, \\
S\left(t_{1}^{+}\right) & <0, \quad t^{+}=\left\{t: t \geq t_{1}\right\}
\end{aligned}
$$

Based on (16) and the first equation of system (6) we have

$$
\left.{ }_{t_{0}}^{c} D_{t_{1}}^{\alpha} S\left(t_{1}\right)\right|_{S\left(t_{1}\right)=0}=0 \quad \text { where } \quad 0<\alpha<1
$$

Using Lemma (1), we get $S\left(t_{1}^{+}\right)=0$, which is a contradiction as $S\left(t_{1}^{+}\right)<0$. Hence $S(t) \geq 0$ for all $t \geq t_{0}$. In similar way we can get $I(t) \geq 0, Y(t) \geq 0$ for all $t \geq t_{0}$.

Now to prove the boundedness of all the solution of system initiated in the region $\mathcal{B}_{+}$, we define the function $V(t)=S(t)+I(t)+Y(t)$, then we have,

$$
\begin{aligned}
& { }_{t_{0}}^{c} D_{t}^{\alpha} V(t)+\eta V(t) \\
& =r_{1} S\left(1-\frac{S+I}{k}\right)\left(\frac{1}{1+f Y}\right)-S I-\frac{p_{1} Y S}{m Y+S} \\
& +S I-\frac{c_{1} Y I}{m Y+I}-\gamma_{1} I+\delta_{1} Y\left(1-\frac{h Y}{S+I}\right)+\eta S \\
& +\eta I+\eta Y \\
& \leq r_{1} S-\frac{r_{1}}{k} S(S+I)-\frac{p_{1} Y S}{m Y+S}-\frac{c_{1} Y I}{m Y+I}-\gamma_{1} I \\
& +\delta_{1} Y-\frac{\delta_{1} h Y^{2}}{I+S}+\eta S+\eta I+\eta Y \\
& \leq\left(r_{1}+\eta\right) S-\frac{r_{1}}{k} S^{2}+Y\left(\delta_{1}+\eta\right)-\frac{\delta_{1} h}{2 \Psi} Y^{2}+\left(\eta-\gamma_{1}\right) I \\
& \leq\left(r_{1}+\eta\right) S-\frac{r_{1}}{k} S^{2}+Y\left(\delta_{1}+\eta\right)-\frac{\delta_{1} h}{2 \Psi} Y^{2} \\
& \leq-r_{1}\left(S-\frac{k\left(r_{1}+\eta\right)}{2 r_{1}}\right)^{2}+\frac{k\left(r_{1}+\eta\right)^{2}}{4 r_{1}}-\frac{\delta_{1} h}{2 \Psi} \\
& \left(Y-\frac{\Psi\left(\delta_{1}+\eta\right)}{\delta_{1} h}\right)^{2}+\frac{\Psi\left(\delta_{1}+\eta\right)^{2}}{2 \delta_{1} h} \\
& \leq \frac{k\left(r_{1}+\eta\right)^{2}}{4 r_{1}}+\frac{\Psi\left(\delta_{1}+\eta\right)^{2}}{2 \delta_{1} h} \\
& \text { where } \eta=\gamma_{1} . \text { By Lemma } 3 \text { we have, }
\end{aligned}
$$

$$
\begin{aligned}
V(t) & \leq\left(V\left(t_{0}\right)-\frac{k\left(r_{1}+\eta\right)^{2}}{4 r_{1}}-\frac{\Psi\left(\delta_{1}+\eta\right)^{2}}{2 \delta_{1} h}\right) \\
& E_{\alpha}\left[-\eta\left(t-t_{0}\right)^{\alpha}\right]+\frac{k\left(r_{1}+\eta\right)^{2}}{4 r_{1}}+\frac{\Psi\left(\delta_{1}+\eta\right)^{2}}{2 \delta_{1} h} \\
& \rightarrow \frac{k\left(r_{1}+\eta\right)^{2}}{4 r_{1}}+\frac{\Psi\left(\delta_{1}+\eta\right)^{2}}{2 \delta_{1} h}, t \rightarrow \infty
\end{aligned}
$$

Hence, all the solution of the fractional-order system (6) which initiate in $\mathcal{B}_{+}$are restricted to the region $\Gamma$, where

$$
\Gamma=\left\{\begin{array}{c}
(S, I, Y) \in \overline{\Omega_{+}} \mid S+I+Y \leq \frac{k\left(r_{1}+\eta\right)^{2}}{4 r_{1}} \\
+\frac{\Psi\left(\delta_{1}+\eta\right)^{2}}{2 \delta_{1} h}+\epsilon, \epsilon>0
\end{array}\right\}
$$

this completes the proof of theorem.

\section{Equilibrium points}

The fractional-order system (6) has the following biologically feasible equilibrium points.

(1) The trivial or vanishing equilibrium $E_{1}(0,0,0)$ which always exists. In an ecological sense, trivial equilibrium is important since all populations will never become extinct simultaneously.

(2) The axial equilibrium $E_{2}(k, 0,0)$ where there is only susceptible prey, which always exists. 
(3) The disease-free equilibrium $E_{3}\left(S_{3}, 0, Y_{3}\right)$ where $S_{3}=\frac{h k\left(r_{1}(h+m)-p_{1}\right)}{f k p_{1}+h r_{1}(h+m)}, Y_{3}=$ $\frac{k\left(r_{1}(h+m)-p_{1}\right)}{f p_{1}+h r_{1}(h+m)}$.

The disease-free equilibrium $E_{3}$ exists if and only if $r_{1}(h+m)>p_{1}$.

(4) The predator-free equilibrium $E_{4}\left(S_{4}, I_{4}, 0\right)$ where $S_{4}=\gamma_{1}, I_{4}=\frac{r_{1}\left(k-\gamma_{1}\right)}{k+r_{1}}$ which exists if and only if $\Re_{0}>1$ where $\Re_{0}=\frac{k}{\gamma_{1}}$. $\Re_{0}$ is the basic reproduction number of the epidemic theory determined with help of next generation matrix method 41.

(5) The positive or endemic equilibrium $E^{*}\left(S^{*}, I^{*}, Y^{*}\right)$ : From the equation of predator nullcline we obtain $Y=\frac{S+I}{h}$. Solving susceptible prey and infected prey nullcline equations and substituting $Y^{*}$ gives,

$$
\begin{gathered}
I^{*}=\frac{c_{1} S^{*}-m\left(S^{*}\right)^{2}+\gamma_{1} m S^{*}}{-c_{1}-\gamma_{1} h+h S^{*}-\gamma_{1} m+m S^{*}}, \\
Y^{*}=\frac{S^{*}\left(S^{*}-\gamma_{1}\right)}{(h+m)\left(S^{*}-\gamma_{1}\right)-c_{1}} .
\end{gathered}
$$

Substituting $I^{*}$ and $Y^{*}$ in susceptible prey nullcline equation gives the following fifth degree polynomial equation,

$$
\begin{aligned}
\rho_{0} S^{*^{5}} & +\rho_{1} S^{*^{4}}+\rho_{2} S^{*^{3}} \\
& +\rho_{3} S^{*^{2}}+\rho_{4} S^{*}+\rho_{5}=0
\end{aligned}
$$

where $\rho_{0}, \rho_{1}, \rho_{2}, \rho_{3}, \rho_{4}$ and $\rho_{5}$ are given in the appendix.

$I^{*}$ and $Y^{*}$ are uniquely defined if $S^{*}$ is a solution of the above equation (20). If $S^{*} \neq 0$ is a real positive solution of the polynomial equation (20), then $I^{*}$ and $Y^{*}$ are real and positive if,

$\frac{c_{1}+\gamma_{1} h+\gamma_{1} m}{h+m}<S^{*}<\frac{c_{1}+\gamma_{1} m}{m}$.

For the parameters provided in section 9 with $f=0.05, m=5, h=0.5, k=350$ equation (20) has a unique non-zero positive real root $S^{*}=41.59$ for which the corresponding $I^{*}=50.57$ and $Y^{*}=164.31$.

\section{Local stability analysis}

Throughout this section, we investigate the local stability of the equilibrium points of the fractional-order system (6). For local stability analysis of the positive equilibrium, we use the Routh-Hurwitz criterion.

\subsection{Local stability of $E_{1}(0,0,0)$}

The Jacobian matrix of the fractional-order system (6) is not well-defined at the equilibrium point $E_{1}(0,0,0)$. In order to show that $E_{1}$ is unstable, it is sufficient to prove that not all the trajectories initiated in the neighborhood of $E_{1}$ approach $E_{1}$. Suppose a trajectory which initiated with $Y(0)=0$ and $S(t)>0$, then we have $Y(t)=0$ but $S(t)>0 \forall t$. Hence

$$
\begin{gathered}
\frac{1}{S} \cdot{ }_{t}^{c} D_{t}^{\alpha} S(t)=r_{1}\left(1-\frac{S+I}{k}\right)-I>\frac{r_{1}}{2} \\
\Longrightarrow{ }_{t}^{c} D_{t}^{\alpha} S(t)>S\left(\frac{r_{1}}{2}\right)
\end{gathered}
$$

Now, if $S$ and $I$ are small enough then by using Lemma 4,

$$
S(t)>S_{0} E_{\alpha}\left[\frac{r_{1}}{2}\left(t-t_{0}\right)\right]
$$

for $t \geq t_{0}$. Therefore the trajectory cannot approaches to $E_{1}$. Hence $E_{1}$ is locally asymptotically unstable.

\subsection{Local stability of $E_{2}(k, 0,0)$}

The Jacobian matrix of the fractional-order system (6) is not well defined at the axial equilibrium $E_{2}(k, 0,0)$. To prove that $E_{2}$ is unstable we presume that $E_{2}$ is locally asymptotically stable (LAS). Now suppose a trajectory which is initiated with $Y_{0}>0$ and either $I_{0}>0$ or $S_{0}>0$. Hence either $I_{t}>0$ or $S_{t}>0 \forall t$. Therefore

$$
{ }_{t}^{c} D_{t}^{\alpha} Y(t) \geq \frac{\delta_{1} Y}{2}
$$

Then by using Lemma 4

$$
Y(t) \geq Y_{0} E_{\alpha}\left[\frac{\delta_{1}}{2}\left(t-t_{0}\right)\right]
$$

for $t \geq t_{0}$. Therefore the trajectory never approaches to $E_{2}$. Hence $E_{2}$ is locally asymptotically unstable.

\subsection{Local stability of $E_{3}\left(S_{3}, 0, Y_{3}\right)$}

Theorem 3. If $\Re_{0}<1+\frac{c_{1}+\chi}{\gamma_{1} m}$ then the diseasefree equilibrium $E_{3}$ of the fractional-order system (6) is locally asymptotically stable under the condition $2 \delta_{1}>\frac{p_{1}}{m}$ and $r_{1}>\frac{p_{1}}{m}$.

Proof. The Jacobian matrix of system (6) at $E_{3}$ is given by

$$
J_{E_{3}}=\left(\begin{array}{ccc}
J_{11} & J_{12} & J_{13} \\
0 & J_{22} & 0 \\
J_{31} & J_{32} & J_{33}
\end{array}\right)
$$

where $J_{11}=\frac{h\left((2 h+f k+m) p_{1}-(h+m)^{2} r_{1}\right)}{(h+f k)(h+m)^{2}}$, $J_{12}=-\frac{h\left((h+m) r_{1}-p_{1}\right)\left(f k p_{1}+(h+m)\left(k(h+f k)+h r_{1}\right)\right)}{(h+f k)(h+m)\left(f k p_{1}+h(h+m) r_{1}\right)}$, 
$J_{13}=-\frac{h p_{1}\left(\left(h^{2}+2 f k h+f k m\right) r_{1}-f k p_{1}\right)}{(h+f k)(h+m)^{2} r_{1}}$,

$J_{22}=\frac{h(h+m) r_{1}\left(m\left(k-\gamma_{1}\right)-c_{1}\right)-k p_{1}\left(f c_{1}+m\left(h+f \gamma_{1}\right)\right)}{m\left(f k p_{1}+h(h+m) r_{1}\right)}$,

$J_{31}=\frac{\delta_{1}}{h}, J_{32}=\frac{\delta_{1}}{h}, J_{33}=-\delta_{1}$.

The eigenvalues of the Jacobian matrix of the system at the equilibrium point $E_{3}$ are the roots of the following equation

$$
\left(J_{22}-\sigma_{I}\right)\left(\sigma^{2}-A \sigma+B\right)=0
$$

where

$A=\frac{h p_{1}(f k+2 h+m)-(h+m)^{2}\left(\delta_{1}(f k+h)+h r_{1}\right)}{(h+m)^{2}(f k+h)}$,

$B=\frac{\delta_{1}\left(r_{1}(h+m)-p_{1}\right)\left(f k p_{1}+h r_{1}(h+m)\right)}{r_{1}(h+m)^{2}(f k+h)}$.

The characteristic equation have the following roots,

$$
\begin{aligned}
\sigma_{I} & =\frac{h r_{1}(h+m)\left(m\left(k-\gamma_{1}\right)-c_{1}\right)-k p_{1}\left(c_{1} f+m\left(\gamma_{1} f+h\right)\right)}{m\left(f k p_{1}+h r_{1}(h+m)\right)} \\
\sigma_{2} & =\frac{h p_{1}(f k+2 h+m)-(h+m)^{2}\left(\delta_{1}(f k+h)+h r_{1}\right)}{2(h+m)^{2}(f k+h)} \\
& -\frac{\hat{\Lambda}}{2 \sqrt{r_{1}}(h+m)^{2}(f k+h)} \\
\sigma_{3} & =\frac{h p_{1}(f k+2 h+m)-(h+m)^{2}\left(\delta_{1}(f k+h)+h r_{1}\right)}{2(h+m)^{2}(f k+h)} \\
& +\frac{\hat{\Lambda}}{2 \sqrt{r_{1}}(h+m)^{2}(f k+h)} \\
\hat{\Lambda} & =\sqrt{-2 p_{1} r_{1}(h+m)^{2}\left(\begin{array}{c}
h^{2} r_{1}(f k+2 h+m) \\
+(f k+h)(f k(3 h+2 m)-h m)
\end{array}\right)} \\
& \times p_{1}^{2}\left(h^{2} r_{1}(f k+2 h+m)^{2}+4 \delta_{1} f k(h+m)^{2}(f k+h)\right) \\
& +r_{1}(h+m)^{4}\left(h r_{1}-\delta_{1}(f k+h)\right)^{2}
\end{aligned}
$$

If $\Re_{0}<1+\frac{c_{1}+\chi}{\gamma_{1} m}$ then $\left|\arg \left(\sigma_{I}\right)\right|=\pi>\frac{\alpha \pi}{2}$ where $\chi=\frac{k p_{1}\left(c_{1} f+m\left(\gamma_{1} f+h\right)\right)}{h r_{1}(h+m)}$.

The eigenvalues $\sigma_{2,3}$ have negative real parts if $2 \delta_{1}>\frac{p_{1}}{m}$ and $\left|\arg \left(\sigma_{2,3}\right)\right|=\pi>\frac{\alpha \pi}{2}, \forall 0<\alpha<1$. Therefore according to Matignon's condition [42], the disease-free equilibrium $E_{3}$ is locally asymptotically stable if $\Re_{0}<1+\frac{c_{1}+\chi}{\gamma_{1} m}$ with the condition that $2 \delta_{1}>\frac{p_{1}}{m}$ and $r_{1}>\frac{p_{1}}{m}$.

\subsection{Local stability of $E_{4}\left(S_{4}, I_{4}, 0\right)$}

Theorem 4. The predator-extinction equilibrium point $E_{4}$ of the system (6) is locally asymptotically unstable if $\Re_{0}>1$.

Proof. The predator-free equilibrium $E_{4}$ exists for $\Re_{0}>1$. The Jacobian matrix of system (6) at $E_{4}$ is given by

$J_{E_{4}}=\left(\begin{array}{ccc}-\frac{r_{1} \gamma_{1}}{k} & -\frac{\left(k+r_{1}\right) \gamma_{1}}{k} & \frac{f r_{1} \gamma_{1}\left(\gamma_{1}-k\right)-p_{1}\left(k+r_{1}\right)}{k+r_{1}} \\ \frac{r_{1}\left(k-\gamma_{1}\right)}{k+r_{1}} & 0 & -c_{1} \\ 0 & 0 & \delta_{1}\end{array}\right)$

The eigenvalues of the Jacobian matrix of the system at $E_{4}$ are the roots of the following equation

$$
\left(\delta_{1}-\Lambda_{Y}\right)\left(\Lambda^{2}+\frac{r_{1} \gamma_{1}}{k}+\frac{\gamma_{1} r_{1}\left(k-\gamma_{1}\right)}{k}\right)=0
$$

Clearly one eigenvalue of the characteristic polynomial is $\Lambda_{Y}=\delta_{1}$. Therefore the system (6) is locally asymptotically unstable at $E_{4}$.

\subsection{Local stability of $E^{*}$}

The Jacobian matrix of system (6) at $E^{*}$ is given by

$$
J_{E^{*}}=\left(\begin{array}{ccc}
V_{1} & V_{2} & V_{3} \\
V_{4} & V_{5} & V_{6} \\
V_{7} & V_{8} & V_{9}
\end{array}\right)
$$

where

$$
\begin{aligned}
V_{1} & =\frac{r_{1}\left(k-I^{*}-2 S^{*}\right)}{f k Y^{*}+k} \\
& -\frac{m p_{1}\left(Y^{*}\right)^{2}+I^{*}\left(m Y^{*}+S^{*}\right)^{2}}{\left(m Y^{*}+S^{*}\right)^{2}} \\
V_{2} & =-\frac{S^{*}\left(f k Y^{*}+k+r_{1}\right)}{f k Y^{*}+k} \\
V_{3} & =S^{*}\left(\frac{f r_{1}\left(-k+I^{*}+S^{*}\right)}{k\left(f Y^{*}+1\right)^{2}}-\frac{p_{1} S^{*}}{\left(m Y^{*}+S^{*}\right)^{2}}\right) \\
V_{4} & =I^{*} \\
V_{5} & =\frac{\left(S^{*}-\gamma_{1}\right)\left(m Y^{*}+I^{*}\right)^{2}-c_{1} m\left(Y^{*}\right)^{2}}{\left(m Y^{*}+I^{*}\right)^{2}} \\
V_{6} & =-\frac{c_{1}\left(I^{*}\right)^{2}}{\left(m Y^{*}+I^{*}\right)^{2}} \\
V_{7} & =\frac{\delta_{1} h\left(\hat{Y}^{*}\right)^{2}}{\left(I^{*}+S^{*}\right)^{2}} \\
V_{8} & =\frac{\delta_{1} h\left(Y^{*}\right)^{2}}{\left(I^{*}+S^{*}\right)^{2}} \\
V_{9} & =\frac{\delta_{1}\left(-2 h Y^{*}+I^{*}+S^{*}\right)}{I^{*}+S^{*}}
\end{aligned}
$$

and the corresponding characteristic equation is of the form

$$
\sigma^{3}+\omega_{1} \sigma^{2}+\omega_{2} \sigma+\omega_{3}=0
$$

where $\omega_{i},(i=1,2,3)$ are given in the Appendix. By Routh-Hurwitz stability criterion the positive equilibrium $E^{*}$ will be locally asymptotically stable if $\omega_{1}>0, \omega_{3}>0$ and $\omega_{1} \omega_{2}>\omega_{3}$.

\section{Global stability analysis}

In this section, we study the global asymptotic stability of the disease-free equilibrium point and the positive equilibrium point by constructing suitable Lyapunov functions. 
Theorem 5. If $\Re_{0}<k\left(S_{3}-\frac{c_{1}}{m}\right)^{-1}$ then the disease-free equilibrium $E_{3}$ is globally asymptotically stable.

Proof. Proof of the theorem is given in the Appendix.

Theorem 6. The positive equilibrium $E^{*}$ is globally asymptotically stable with respect to solutions initiating in the interior of the region $\Gamma$ if $L_{2}=L_{1}\left(1+\frac{r_{1}}{k\left(1+f Y^{*}\right)}\right)$ and $\frac{r_{1}}{k\left(1+f Y^{*}\right)}>\frac{p_{1}}{m^{2} Y^{*}}$.

Proof. Proof of the theorem is given in the Appendix.

\section{Bifurcation analysis}

Throughout this section, we analyze the possibility of occurrence of Hopf-bifurcation at the disease-free equilibrium point $E_{3}$ and positive equilibrium $E^{*}$. Oscillating behavior is one of the most frequent dynamical behavior appears in the nonlinear mathematical study of population dynamics, which lead to the Hopf-bifurcation of the system.

From the equation (21), the characteristic equation of the Jacobian matrix of the system at $E_{3}$ has a pair of purely imaginary eigenvalues for $A=0$ and $B>0$ which implies,

$$
\begin{aligned}
r_{1} & >\frac{2 h p_{1}+m p_{1}}{h^{2}+2 h m+m^{2}} \\
f & >\frac{h^{2} r_{1}+2 h m r_{1}-2 h p_{1}+m^{2} r_{1}-m p_{1}}{k p_{1}} \\
c_{1} & >\frac{m\left(h r_{1}(h+m)\left(k-\gamma_{1}\right)-k p_{1}\left(\gamma_{1} f+h\right)\right)}{f k p_{1}+h r_{1}(h+m)} \\
\delta_{1} & =\frac{h\left(p_{1}(f k+2 h+m)-r_{1}(h+m)^{2}\right)}{(h+m)^{2}(f k+h)}
\end{aligned}
$$

Since we are discussing the effect of fear for the model, so we use rate of fear $f$ as the bifurcation parameter. Again the characteristic equation of $J_{E^{*}}$ is of the form,

$$
\sigma^{3}+\omega_{1} \sigma^{2}+\omega_{2} \sigma+\omega_{3}=0
$$

The positive equilibrium $E^{*}$ experiences a Hopf bifurcation for some free parameter say $f$ at a threshold value $f=f^{*}$ if $\omega_{1}\left(f^{*}\right), \omega_{2}\left(f^{*}\right), \omega_{3}\left(f^{*}\right)>$ $0, \Delta=\omega_{1}\left(f^{*}\right) \omega_{2}\left(f^{*}\right)-\omega_{3}\left(f^{*}\right)=0$ and $\frac{\partial \Delta}{\partial f}\left(f^{*}\right) \neq$ 0 . Next, we mention Matignon's criterion for the existence of a Hopf bifurcation when the order $\alpha$ of the fractional derivative passes through the threshold value $\alpha=\alpha^{*}$.

Theorem 7. [44] (Existence of Hopf bifurcation) When the bifurcation parameter $\alpha$ passes through the critical value $\alpha=\alpha^{*} \in(0,1)$, the fractional-order system (6) undergoes a Hopf bifurcation at any equilibrium point $E$ if the following conditions hold

(a) the Jacobian matrix of the system at the equilibrium point $E$ has a pair of complex conjugate eigenvalues $\hat{\lambda}_{2,3}=u \pm i v$ where $u>0$ and one negative real root $\hat{\lambda}_{1}$.

(b) $\hat{m}\left(\alpha^{*}\right)=\alpha^{*} \frac{\pi}{2}-\min _{1 \leq i \leq 3}\left|\arg \left(\hat{\lambda}_{i}\right)\right|=0$.

(c) $\left.\frac{d \hat{m}(\alpha)}{d \alpha}\right|_{\alpha=\alpha^{*}} \neq 0$ (transversality condition)

\section{Numerical simulation}

Throughout this section, we compare the analytical findings using a biologically plausible parameter set. Approximate solutions for our fractionalorder system are determined using the generalized Adams-Bashforth-Moulton type predictorcorrector scheme 43. We took the majority of our base parameter values from the ecoepidemiological study of pelicans in the Salton sea by Chattopadhyay et al. [35].

$$
\begin{aligned}
& r=3 / \text { day }, \quad c=0.05 / \text { day }, \quad \gamma=0.24 / \text { day } \\
& \delta=0.09 / \text { day }, \quad \lambda=0.006 / \text { day }, \quad m=1
\end{aligned}
$$

Additionally, we take $f=0.2, p=0.03 /$ day, $h=0.2$.

With these parameter values,

$$
\begin{aligned}
& r_{1}=\frac{3}{0.006}, f=0.5, p_{1}=\frac{0.03}{0.006}, m=1, \\
& c_{1}=\frac{0.05}{0.006}, \gamma_{1}=\frac{0.24}{0.006}, \delta_{1}=\frac{0.09}{0.006}, h=0.2 .
\end{aligned}
$$

Now, we fix total capacity of the prey to be $k=75$. For the choice of parameter values mentioned above, $S_{3}=45.76$ and $\left(\frac{c_{1}}{m}+\gamma_{1}\right)=48.33$ satisfying $S_{3}-\frac{c_{1}}{m}-\gamma_{1}<0$. Equivalently, $\Re_{0}<$ $k\left(S_{3}-\frac{c_{1}}{m}\right)^{-1}$, which is the condition for global stability of $E_{3}$ obtained analytically in Theorem 5. With these set of parameter values the equilibrium points of the fractional-order system (6) are

$$
E_{3}=(45.7692,0,228.846), E_{4}=(40 ., 30.4348,0)
$$

Under the above parameters no positive equilibrium appears. Between the two equilibria, $E_{4}$ is unstable (Theorem 4) and Theorem 5 is satisfied for global asymptotic stability of $E_{3}$. It is observed that all the trajectories of the system (6) initialed at different values approach to the disease-free equilibrium $E_{3}$, see Figure 1. Next, we consider $f=0.05, m=5, h=0.5$ and $k=350$ along with the other parameters mentioned above. For these parameters $S_{3}=328.46$, $\left(\frac{c_{1}}{m}+\gamma_{1}\right)=41.66$ and $S_{3}-\frac{c_{1}}{m}-\gamma_{1}>0$ which is equivalently $\Re_{0}>k\left(S_{3}-\frac{c_{1}}{m}\right)^{-1}$. The equilibrium 
points of the fractional-order system (6) which exist under these parameters are

$E_{2}=(350,0,0), \quad E_{3}=(328.462,0,656.923)$,

$E_{4}=(40,182.353,0)$,

$E^{*}=(41.5882,40.5682,164.313)$. Among these equilibria, $E_{3}$ is unstable since the Theorem 5 is not satisfied for the parameter set. From numerical simulations, it is found that trajectories of the system tend to the positive equilibrium $E^{*}$ with the increase in time irrespective of the initial value (Figure 5). This suggests that the positive equilibrium $E^{*}$ has a large domain of attraction. Again we set the parameter values $h=0.04$, $k=75$ together with $p_{1}, m, \gamma_{1}, \delta_{1}$ as mentioned in the beginning of section 9 . From the first condition in equation (23),

$$
r_{1}>4.9926
$$

So we fix $r_{1}=\frac{1}{0.006}$, then from the second condition $f>0.466311$. Again we fix $f=0.5$, then from third condition $c_{1}>1.59729$, so we fix $c_{1}=\frac{0.05}{0.006}$. Finally for the fourth condition $\delta_{1}=0.0124456$ which implies $\delta \rightarrow 0$, i.e., the reproduction rate of predator population becomes very very small. For $r_{1}, c_{1}, \delta_{1}$ as above, keeping other parameter values fixed with $f=0.6$ and initial population $(55,75,190)$, the eigenvalues of the Jacobian matrix of the system at the disease-free equilibrium $E_{3}$ are $\hat{\lambda}_{1}=-46.156$, $\hat{\lambda}_{2,3}=0.0143594 \pm 0.244213 i$. From the second condition of Theorem 7 .

$$
\alpha^{*}=\frac{2}{\pi} \arctan \left|\frac{0.244213}{0.0143594}\right|=0.962611 \approx 0.962
$$

and from the last condition,

$$
\left.\frac{d \hat{m}}{d \alpha}\right|_{\alpha=\alpha^{*}}=\frac{\pi}{2} \neq 0
$$

which implies that the transversality condition holds. Hence the fractional-order system (6) at the disease-free equilibrium $E_{3}$ experiences a Hopf bifurcation when the bifurcation parameter $\alpha$ passes through a critical value $\alpha^{*} \approx 0.962$, see Figure 2, Our main interest is to discuss the fear induced in the prey as an anti-predator reaction. We fix the rate of fear $f$ as a free parameter. The system (6) exhibits oscillatory behaviour at the disease-free equilibrium $E_{3}$ for $f=0.7$, $r_{1}=1 / 0.006, c_{1}=0.05 / 0.006, \delta_{1}=0.0124456$ and $\alpha=0.98$, see Figure 3 . We take the initial population $(55,75,190)$ and $\alpha=0.98$ with the fear coefficient $f$ as a free parameter. It is observed that increasing the fear effect $f$ from $f=$ 0.466311 the disease-free equilibrium $E_{3}$ becomes unstable due to a Hopf bifurcation when the bifurcation parameter $f$ passes through a critical value $f^{*}=0.48$, see Figure 4. Again we set the parameter set $f=0.05, m=5, h=0.5, k=350$ together with other parameter values as mentioned in the beginning of section 9. In Figure 5, we plot the trajectories of the system (6) for $\alpha=0.99$ with initial population $(55,170,450)$ which approach to $E^{*}(41.59,40.57,164.31)$. Moreover, for these parameter values $\omega_{1}>0, \omega_{3}>0$ and $\omega_{1} \omega_{2}-\omega_{3}>0$, which confirms that the Routh-Hurwitz stability criterion satisfies. 


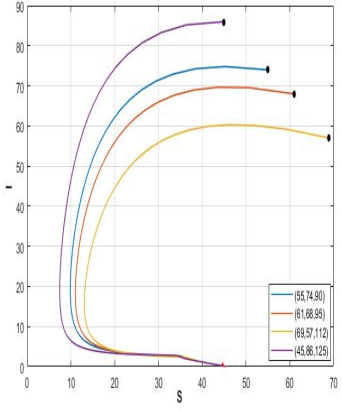

(a)

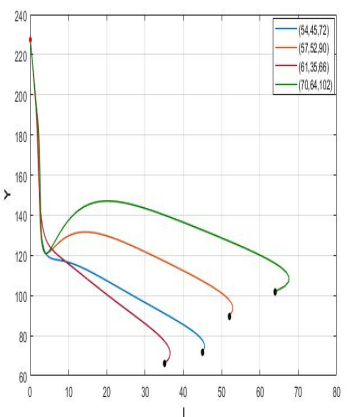

(c)

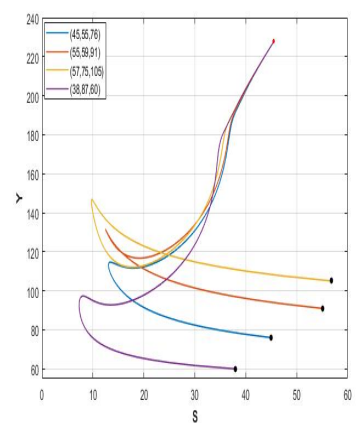

(b)

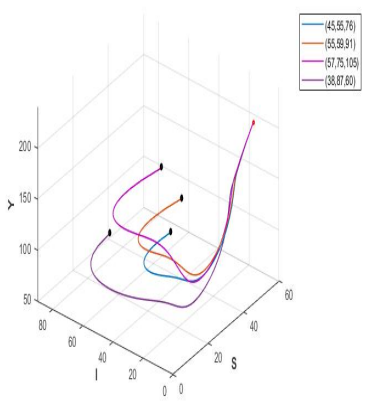

(d)
Figure 1. Phase diagram of the system (6) at the disease-free equilibrium $E_{3}$ with different initial values with $\alpha=0.9$.

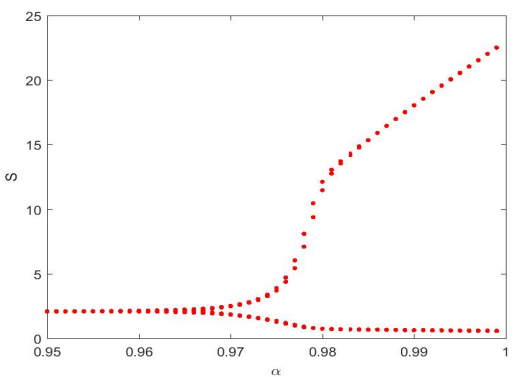

(a)

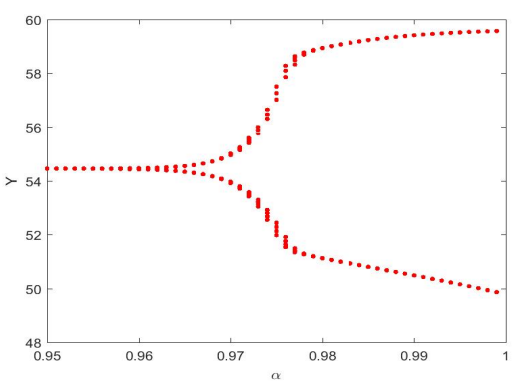

(b)

Figure 2. Bifurcation diagram of the system at $E_{3}$ with respect to the bifurcation parameter $\alpha$.

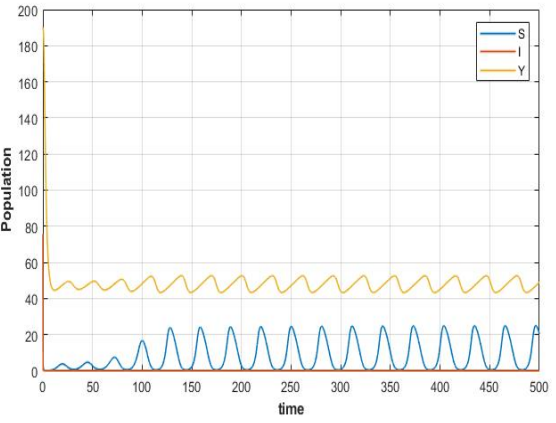

Figure 3. Time series of the fractional-order system (6) at $E_{3}$ for $f=0.7$ and $\alpha=0.98$

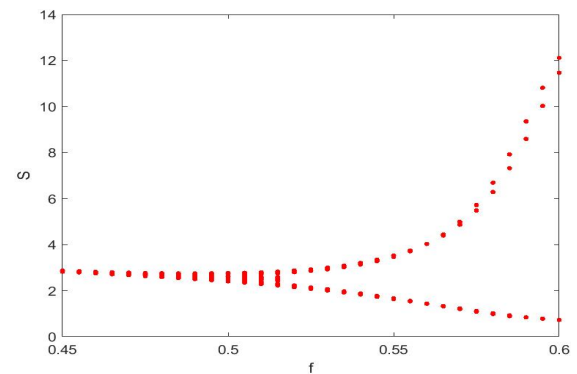

(a)

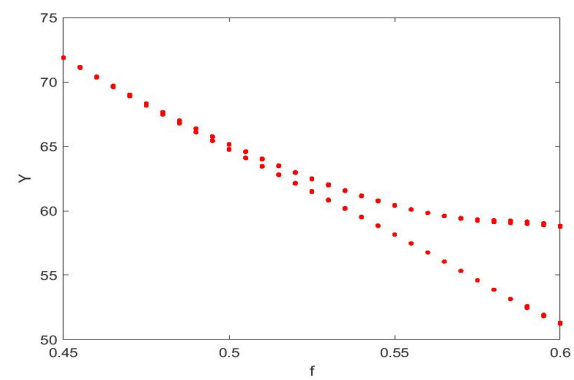

(b)

Figure 4. Bifurcation diagram of the system at $E_{3}$ with respect to the fear effect $f$ for $\alpha=0.98$

In Figure 6, we compute orbits with above parameters and $\alpha=0.99$ from several starting points and observe that all trajectories of the system (6) approach to the same positive equilibrium $E^{*}(41.59,40.57,164.31)$. This suggests that $E^{*}$ has a large domain of attraction. We fix the parameter values $f=0.05, m=5, h=0.5, k=350$ together with the parameter values mentioned in the beginning of section 9 and keep $\gamma_{1}$ as free parameter. For $\gamma_{1}=\frac{0.12}{0.006}$ and $\alpha=1$ it is observed that for all the trajectories of the system (6) undergoes a Hopf bifurcation, see Figure 7 and Figure 8. Increasing $\gamma_{1}$ from $\gamma_{1}=\frac{0.12}{0.006}$ to $\gamma_{1}=\frac{0.135}{0.006}$, it is observed that the positive equilibrium $E^{*}$ undergoes a backward Hopf bifurcation at $\gamma_{1}=0.1284 / 0.006$, see Figure 9. 


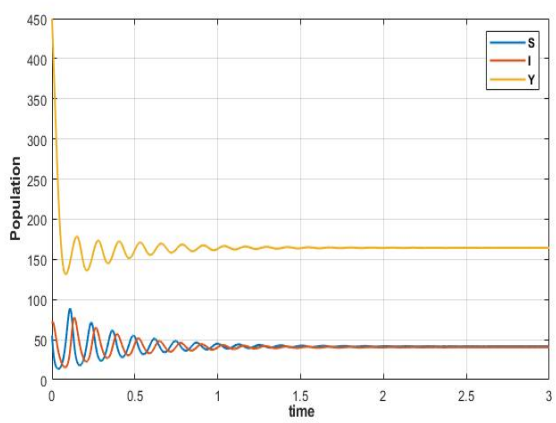

(a)

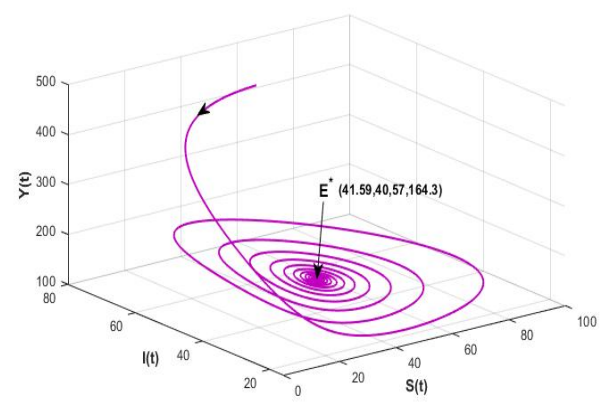

(b)

Figure 5. (a) Time series of positive equilibrium point $E^{*}$ for $\alpha=0.99$, (b) Phase diagram of positive equilibrium point $E^{*}$ for $\alpha=0.99$.

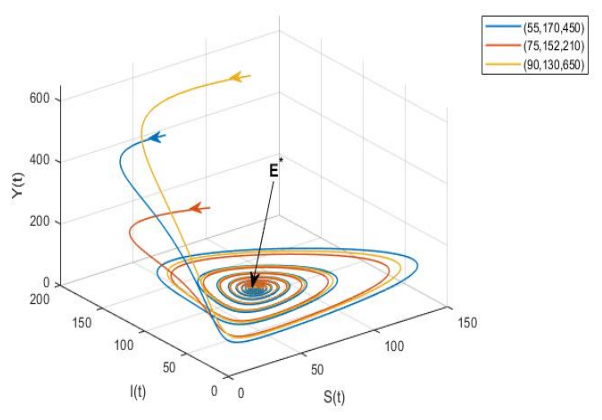

Figure 6. Phase portrait of the positive equilibrium $E^{*}$ of the system (6) for $\alpha=0.99$.

For $\gamma_{1}=\frac{0.07}{0.006}$ the eigenvalues of the Jacobian matrix of the system at the positive equilibrium $E^{*}$ are $\hat{\lambda}_{1}=-19.0552, \hat{\lambda}_{2,3}=0.849491 \pm 32.1521 i$. From Theorem 7 ,

$$
\alpha^{*}=\frac{2}{\pi} \arctan \left|\frac{32.1521}{0.849491}\right|=0.983184 \approx 0.983
$$

and

$$
\left.\frac{d \hat{m}}{d \alpha}\right|_{\alpha=\alpha^{*}}=\frac{\pi}{2} \neq 0
$$

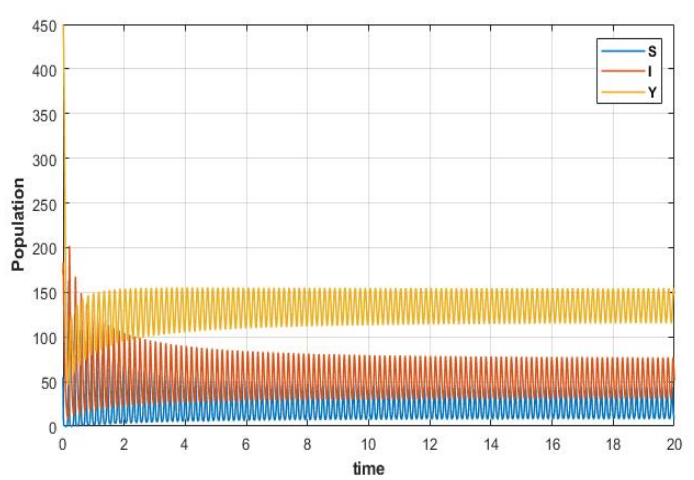

Figure 7. Time series of the fractional-order system (6) for $\gamma_{1}=\frac{0.12}{0.006}$ and $\alpha=1$

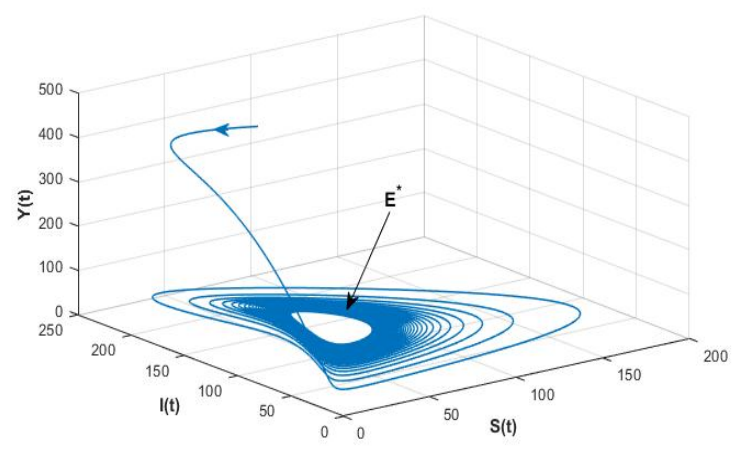

Figure 8. Phase diagram of the fractional-order system (6) for $\gamma_{1}=$ $\frac{0.12}{0.006}$ and $\alpha=1$

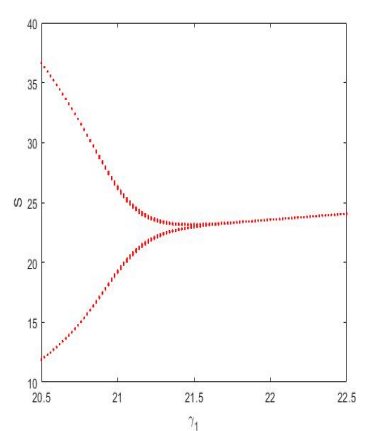

(a)

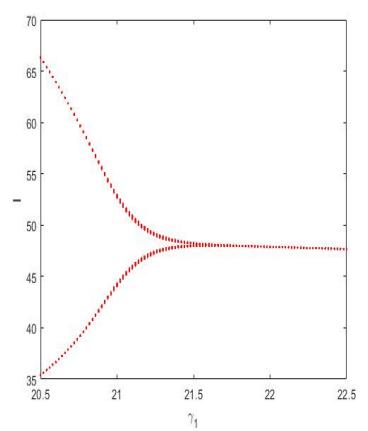

(b)

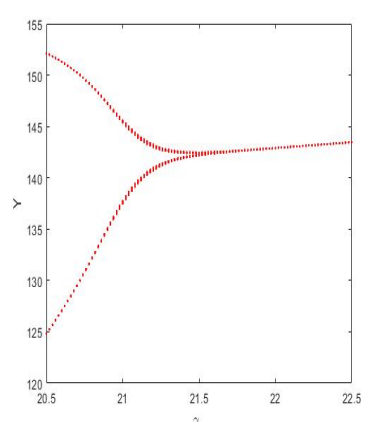

(c)

Figure 9. Bifurcation diagram of the system at $E^{*}$ with respect to mortality rate of infected prey $\gamma_{1}$ for $\alpha=$ 1. 


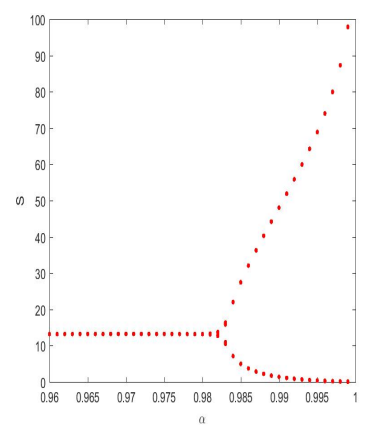

(a)

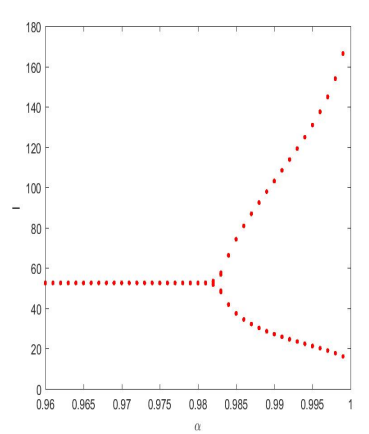

(b)

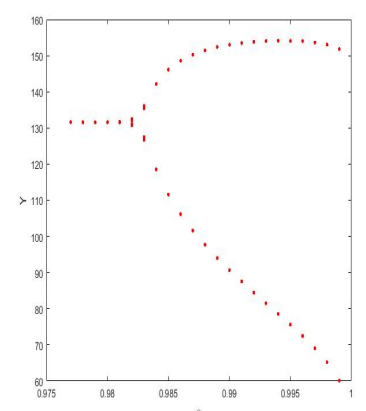

(c)

Figure 10. Bifurcation diagram of the system at $E^{*}$ with respect to the bifurcation parameter $\alpha$.

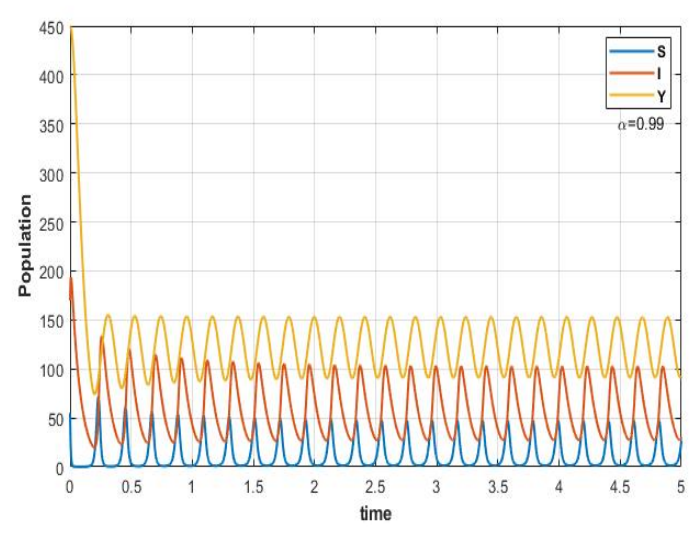

Figure 11. Time series of the system (6) for $\gamma_{1}=\frac{0.07}{0.006} \quad \alpha=0.99$.

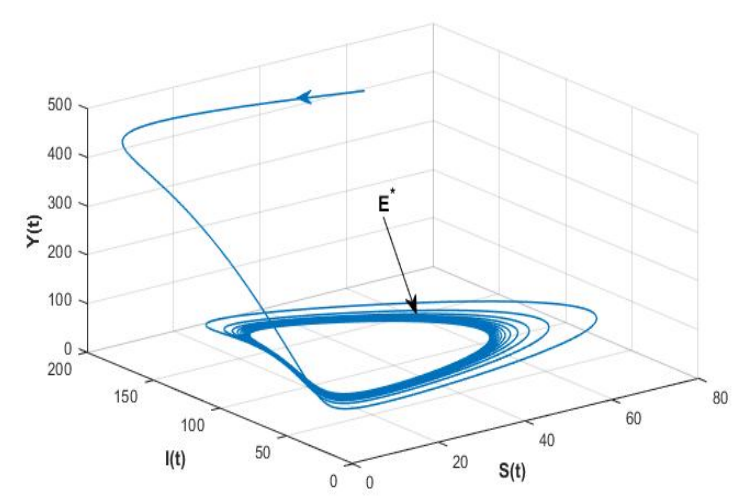

Figure 12. Phase diagram of the system for $\gamma_{1}=\frac{0.07}{0.006}$ and $\alpha=0.99$.
Hence Hopf bifurcation occurs in the system (6) at the positive equilibrium $E^{*}$ when the bifurcation parameter $\alpha$ passes through a critical value $\alpha^{*}=0.983$, see Figure 10 . For $\gamma_{1}=\frac{0.07}{0.006}$ and $\alpha=0.99$ all the trajectories of the system (6) shows oscillatory behaviour via a Hopf bifurcation, see Figure 11 and Figure (12). Increasing $\gamma_{1}$ from $\gamma_{1}=\frac{0.087}{0.006}$ to $\gamma_{1}=\frac{0.099}{0.006}$, it is observed that at the positive equilibrium $E^{*}$ the system (6) undergoes a backward Hopf bifurcation when the bifurcation parameter $\gamma_{1}$ passes through a critical value $\gamma_{1}^{*}=\frac{0.093}{0.006}$, see Figure 13 .

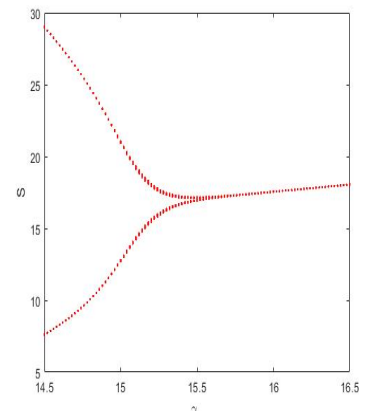

(a)

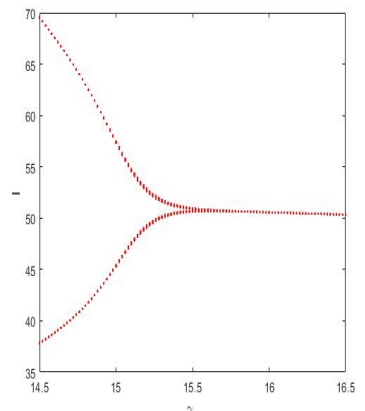

(b)

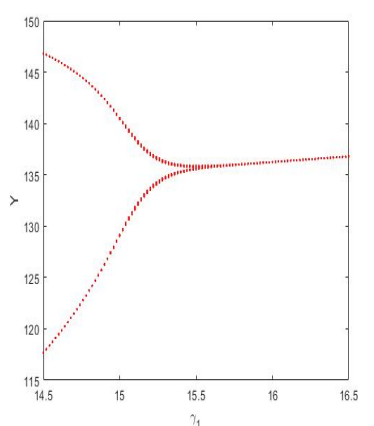

(c)

Figure 13. Bifurcation diagram of the system at $E^{*}$ with respect to mortality rate of infected prey $\gamma_{1}$ for $\alpha=$ 0.99 .

For $h=05, k=2500, f=0.05$ along with other parameters as mentioned in section 9 and initial population $(55,170,450)$ all the population coexists with population $E^{*}(40.93,154.9,39.18)$ (Figure 14). For $f=0.12$ the eigenvalues of the Jacobian matrix of the system at the positive equilibrium $E^{*}$ are $\hat{\lambda}_{1}=-20.8432$, $\hat{\lambda}_{2,3}=2.29661 \pm 70.1413 i$. From Theorem 7 .

$$
\alpha^{*}=\frac{2}{\pi} \arctan \left|\frac{70.1413}{2.29661}\right|=0.979163 \approx 0.979
$$

and

$$
\left.\frac{d \hat{m}}{d \alpha}\right|_{\alpha=\alpha^{*}}=\frac{\pi}{2} \neq 0 .
$$

Hence a Hopf bifurcation occurs in the fractionalorder system (6) at the positive equilibrium $E^{*}$ when the bifurcation parameter $\alpha$ passes through 
a critical value $\alpha^{*}=0.979$, see Figure 15 . With a increase in the fear coefficient all the trajectories of the system (6) at the positive equilibrium $E^{*}$ undergoes a Hopf bifurcation. In Figure 16, the oscillatory behaviour of all the population is presented for $f=0.12$ and $\alpha=0.98$. Increasing $f$ from $f=0.05$ to $f=0.18$, it is observed that the positive equilibrium undergoes a forward Hopf bifurcation when the bifurcation parameter $f$ passes through a critical value $f^{*}=0.1$, see Figure 17 .

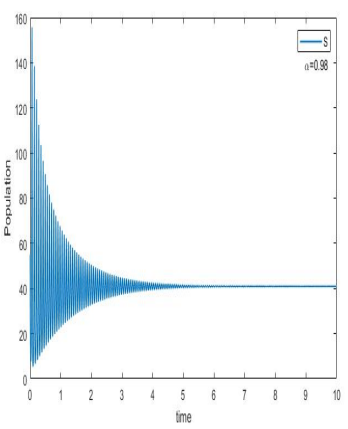

(a)

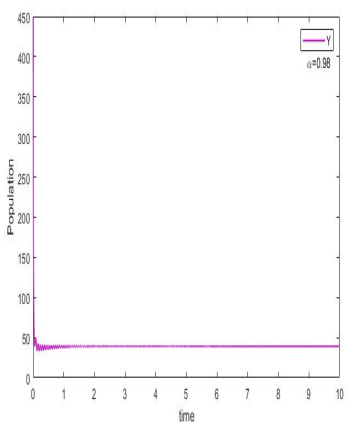

(c)

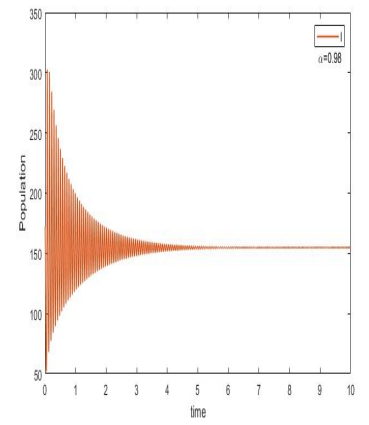

(b)

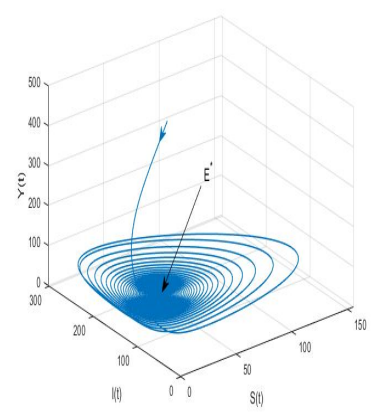

(d)
Figure 14. Time series and phase diagram of the system for $f=0.05, \alpha=0.98$.

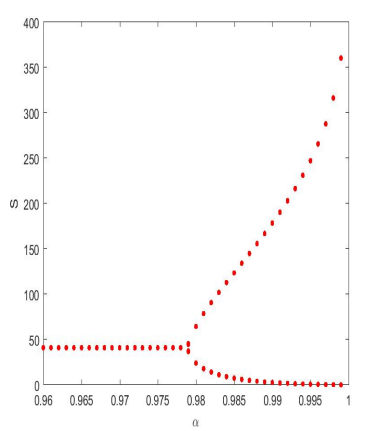

(a)

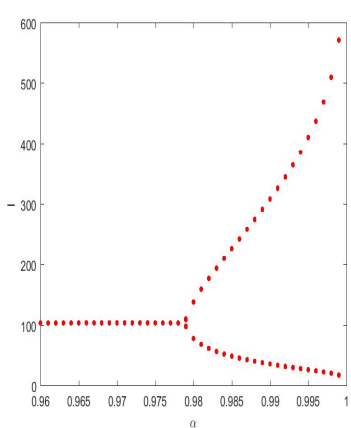

(b)

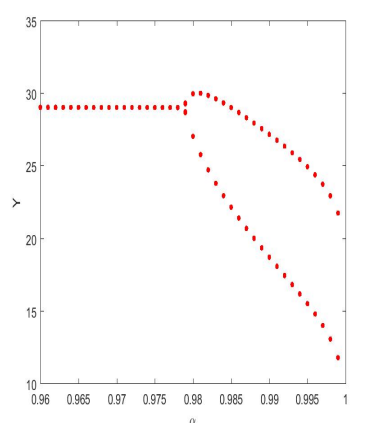

(c)

Figure 15. Bifurcation diagram of the system at $E^{*}$ with respect to the bifurcation parameter $\alpha$.

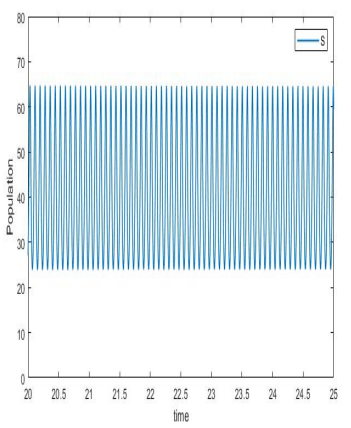

(a)

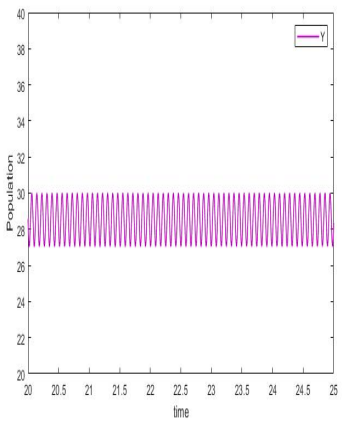

(c)

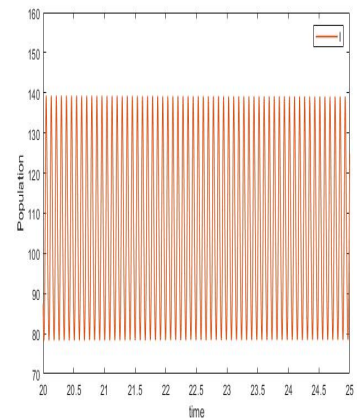

(b)

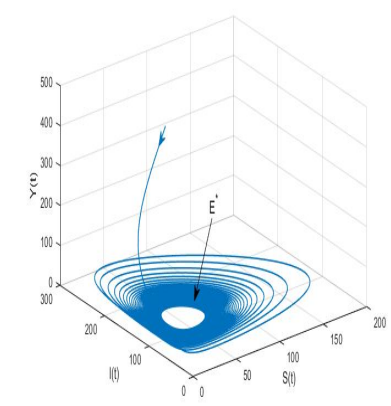

(d)

Figure 16. Time series and phase diagram of the system for $f=0.12$ $\alpha=0.98$. 


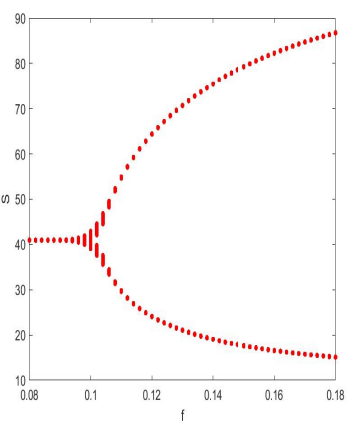

(a)

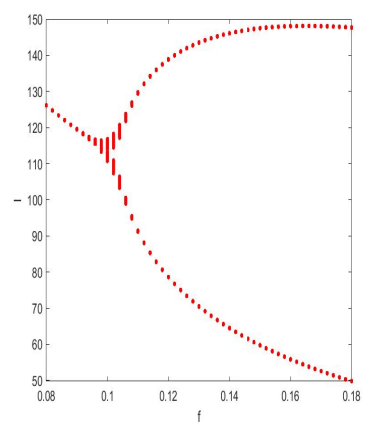

(b)

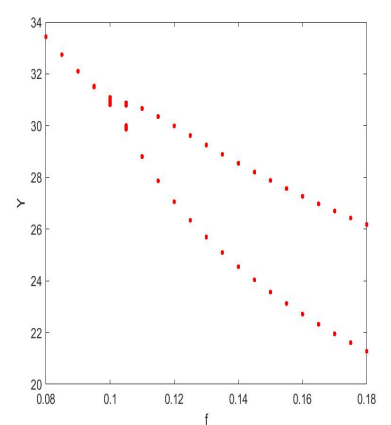

(c)

Figure 17. Bifurcation diagram of the system at $E^{*}$ with respect to to fear coefficient $f$ for $\alpha=0.98$.

\subsection{Impact of the disease on predators in the absence of susceptible prey}

In the absence of susceptible prey both the infected prey and predator populations become extinct, and the system approaches the trivial equilibrium $E_{1}$. Analytical discussion of the situation can be found in literature 37. Here we are exploring this scenario numerically for different values $\alpha$. We fix the parameter values $m=1, c_{1}=\frac{0.03}{0.006}, \gamma_{1}=\frac{0.01}{0.006}, \delta_{1}=\frac{0.05}{0.006}, h=0.2$ and consider other parameters to be 0 . From Figure 18, it is observed both the infected prey and predator populations approach towards extinction for the initial population $(I=75, Y=120)$. The behaviour of the infected prey and predator towards extinction under different values $\alpha$ is presented in Figure 19. Increasing the order $\alpha$, the time duration of extinction for both the species become reduced.

\section{Conclusion and discussion}

In this paper, we investigate a modified ecoepidemiological model incorporating the fear effect. The model equations are constructed with the help of Caputo fractional-order differential equations. Fundamental requisites such as existence, uniqueness, non-negativity, and boundedness of the solutions of the system are discussed.

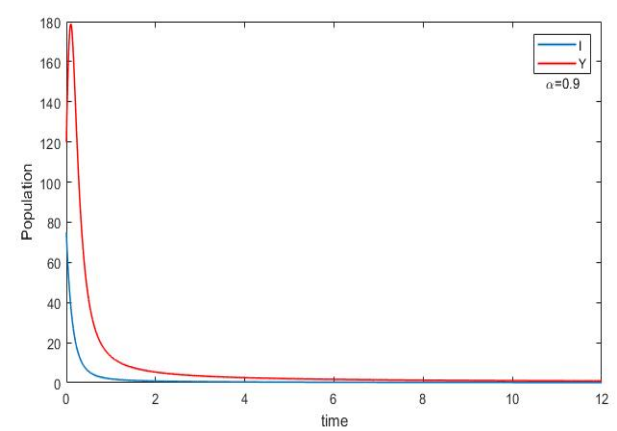

(c)

Figure 18. Time series of the system in absence of susceptible prey for $\alpha=0.9$.

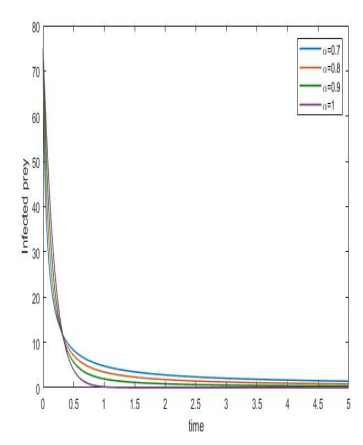

(a)

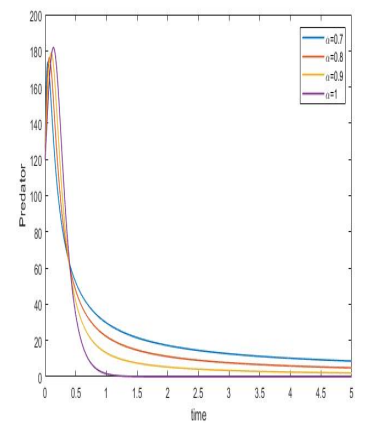

(b)
Figure 19. (a),(b) Time series of the system when $S=0$. Infected prey and predator approach towards extinction for different values of $\alpha$.

Biologically possible equilibrium states of the model are determined. The basic reproduction number $\Re_{0}$ of the epidemiology theory is determined. Local and global asymptotic stabilities of the equilibrium states are presented. The diseasefree equilibrium $E_{3}$ is globally asymptotically stable if $\Re_{0}<k\left(S_{3}-\frac{c_{1}}{m}\right)^{-1}$, where $\Re_{0}$ is the basic reproduction number of the epidemic. The global stability condition of the endemic case, i.e., the positive equilibrium, is also discussed. We determine the threshold parameter values for which the disease-free case and the endemic case become unstable. In equation (23) we present a parametric condition for which the disease-free equilibrium loses its stability due to a Hopf bifurcation. With biologically plausible parameters, we conduct numerical simulations to visualize the system's behaviour near the equilibrium points. To explore the role of the order $(\alpha)$ of the differential equations towards the stability of the equilibrium states, we use Matignon's theorem. Global stability of the system at the disease-free equilibrium $E_{3}$ is presented in Figure 1. The trajectories of the system at the equilibrium $E_{3}$ with $\alpha$ as a free parameter are presented in Figure 
2. Applying Matignon's theorem, we determine the threshold value of $\alpha$ as $\alpha^{*} \approx 0.962$. When $\alpha$ passes through the critical value $\alpha=\alpha^{*}$, the endemic equilibrium $E^{*}$ of the system becomes unstable via a Hopf bifurcation. An increase in the fear coefficient $f$ through a critical value $f^{*}=0.48$ (with $\alpha=0.98$ ), the system shows oscillatory behaviour at the equilibrium $E_{3}$ via a Hopf bifurcation (Figure 4). From an ecological point of view, an increase in fear due to predation risk above a threshold value decreases the prey population's reproduction rate, forcing the system towards extinction. Global asymptotic stability of the positive equilibrium can be observed in Figure 6. Behaviour of the system at $E^{*}$ with respect to $\alpha$ can be seen in Figure 10. Mass mortality of the pelicans was taking place mainly because of consuming infected tilapias in the Salton Sea. Numerically, it is observed that the parameter $\gamma_{1}$ could stabilize the system dynamics at the positive equilibrium when it passes through a critical value $\gamma_{1}=\gamma_{1}^{*}=0.093 / 0.006$ (Figure 13). For a different set of parameters, the system shows oscillatory behaviour through a Hopf bifurcation when $\alpha$ passes through $\alpha=\alpha^{*} \approx 0.979$ (Figure 15). It is also observed that the rate of fear in prey due to predation risk is responsible for the stability of the endemic equilibrium. An increase in the rate of fear due to predation risk enforces the endemic equilibrium to lose its stability via a Hopf bifurcation (Figure 17). In ecological terms, all the populations exhibit oscillatory behaviour with a certain increase in the rate of fear due to predation risk. From the numerical simulations, it is observed that below some threshold value $0<\alpha<\alpha^{*}$ all the population coexists. So, it can be concluded that the system's fraction-order $(\alpha)$ can help to control the coexistence of all the species populations. In the absence of susceptible prey, both infected prey and predator extinct after a specific time. The effect of infected prey on the existence of the predator population is discussed numerically in subsection 9.1. It is observed that with an increase in the order $\alpha$, the time of extinction for both the species get reduced (Figure 18).

We have already given brief summary of the models in 34 37 at the introduction part. As per the authors' information, fewer studies have been done in epidemic models with the fear effect. In [46], Wu et al. studied a delayed epidemic model incorporating fear effect in prey and refuge. Pal investigated a modified LesliGower eco-epidemiological model with fear effect in prey 47] and observed that an increase in the fear coefficient stabilizes the system dynamics. In [49], Sha et al. investigated an ecoepidemiological model with disease in the prey. They assumed that the induced fear also reduces disease transmission along with reproduction and obtained fear-induced backward bifurcation and bi-stability. Our model differs from the model proposed by Sha et al. [49] in functional response, fear effect (no impact of fear effect in disease transmission ), and type of the equations. In a fractional-order sense, Mandal et al. 48 discussed an epidemic model with the fear effect of an infectious disease. Our model is more realistic than the models studied in 35, 37 in the sense of fear induced in prey and the type of the differential equations. The reproduction rate in the prey population is affected because of the predation risk that controls the system dynamics. Again, the fractional-order of the equations may help to control the stability of the coexistence equilibrium state. Interested readers can modify this model by involving non-local, additionally, nonsingular fractional derivatives such as the ABC derivative. Holling type IV functional response can be assumed as most prey shows antipredator defense mechanism. Group defense is a popular anti-predator response where the prey defends themselves by making groups; see 45]. Moreover, one can investigate the model, including the impact of fear in the disease transmission by involving fractional derivatives, see [49].

Conflict of Interests: The authors declare that there is no conflict of interest regarding the publication of this paper.

\section{Acknowledgments}

The authors would like to thank the editor and the referees for their valuable comments and suggestions that improved the quality of our paper.

\section{References}

[1] Zanette L.Y. \& Clinchy, M. (2019). Ecology of fear, Current Biology, 29(9), R309-R313.

[2] Cresswell, W. (2011). Predation in bird populations, Journal of Ornithology, 152(1), 251263.

[3] Fardell, L. L., Pavey, C. R., \& Dickman, C. R. (2020). Fear and stressing in predator-prey ecology: considering the twin stressors of predators and people on mammals. Peer J, 8, e9104. 
[4] Creel, S. \& Christianson, D. (2008). Relationships between direct predation and risk effects. Trends in Ecology 83 Evolution, 23(4), 194-201.

[5] Clinchy, M., Sheriff, M. J. \& Zanette, L.Y. (2013). Predator-induced stress and the ecology of fear. Functional Ecology, 27(1), 56-65.

[6] Zanette, L.Y., White, A.F., Allen, M.C. \& Clinchy, M. (2011). Perceived predation risk reduces the number of offspring songbirds produce per year. Science, 334(6061), 13981401.

[7] Schreck, C.B., Contreras-Sanchez, W. \& Fitzpatrick, M.S. (2001). Effects of stress on fish reproduction, gamete quality, and progeny. Reproductive Biotechnology in Finfish Aquaculture, 3-24.

[8] Abrahams, M.V. \& Sutterlin, A. (1999). The foraging and antipredator behaviour of growth-enhanced transgenic Atlantic salmon. Animal Behaviour, 58(5), 933-942.

[9] Schreck, C.B. (2010). Stress and fish reproduction: the roles of allostasis and hormesis. General and Comparative Endocrinology, 165(3), 549-556.

[10] Hurlbert, A.H., Anderson, T.W., Sturm, K.K. \& Hurlbert, S.H. (2007). Fish and fisheating birds at the Salton Sea: a century of boom and bust. Lake and Reservoir Management, 23(5), 469-499.

[11] Friend, M. (2002). Avian disease at the Salton Sea. In The Salton Sea. Springer, Dordrecht, 293-306

[12] Rocke, T.E., Nol, P., Pelizza, C. \& Sturm, K.K. (2004). Type C botulism in pelicans and other fish-eating birds at the Salton Sea. Studies in Avian Biology, 27, 136-140.

[13] Kermack, W.O. \& McKendrick, A.G. (1927). A contribution to the mathematical theory of epidemics. Proceedings of the royal society of London. Series A, Containing papers of a mathematical and physical character, 115(772), 700-721.

[14] Anderson, R.M. \& May, R.M. (1986). The invasion, persistence, and spread of infectious diseases within animal and plant communities. Philos. Trans. R. Soc. Lond., Ser. B, 314, 533-570.

[15] Hadeler, K.P. \& Freedman, H.I., (1989). Predator-prey populations with parasitic infection. J. Math. Biol., 27, 609-631.

[16] Venturino, E. (1995) Epidemics in predatorprey models: diseases in the prey. Mathematical Population Dynamics: Analysis of Heterogeneity 1 , 381-393.
[17] Chattopadhyay, J. \& Arino, O., (1999). A predator-prey model with disease in the prey. Nonlinear Anal., 36, 747-766.

[18] Wang, X., Zanette, L. \& Zou, X. (2016). Modelling the fear effect in predator-prey interactions. Journal of Mathematical Biology, 73(5), 1179-1204.

[19] Hossain, M., et al.(2020) . Fear Induced Stabilization in an Intraguild Predation Model. International Journal of Bifurcation and Chaos,30(04) , 2050053.

[20] Pal, S., Pal, N., Samanta, S. \& Chattopadhyay, J. (2019). Fear effect in prey and hunting cooperation among predators in a LeslieGower model. Math. Biosci. Eng, 16(5), 51465179.

[21] Panday, P., et al. (2018).Stability and bifurcation analysis of a three-species food chain model with fear. International Journal of Bifurcation and Chaos, 28(01), 1850009.

[22] Li, H.L., et al. (2017) Dynamical analysis of a fractional-order predator-prey model incorporating a prey refuge. Journal of Applied Mathematics and Computing, 54(1-2), 435-449.

[23] Heymans, N. \& Podlubny, I. (2006). Physical interpretation of initial conditions for fractional differential equations with RiemannLiouville fractional derivatives. Rheologica Acta, 45(5), 765-771.

[24] Kilbas, A.A., Srivastava, H.M. \& Trujillo, J.J. (2006). Theory and applications of fractional differential equations, Elsevier,NorthHolland.

[25] Ansari, S.P., Agrawal, S.K. \& Das, S. (2015), Stability analysis of fractional-order generalized chaotic susceptible-infected-recovered epidemic model and its synchronization using active control method. Pramana, 84, 23-32.

[26] Khan, M.A., Ismail, M., Ullah, S. \& Farhan, M. (2020). Fractional order SIR model with generalized incidence rate. AIMS Mathematics, 5(3), 1856-1880.

[27] Caputo, M. \& Fabrizio, M. (2015). A new definition of fractional derivative without singular kernel. Progr. Fract. Differ. Appl., 1(2), $1-13$.

[28] Atangana, A. \& Baleanu, D. (2016). New fractional derivatives with nonlocal and non-singular kernel: theory and application to heat transfer model. arXiv preprint arXiv:1602.03408.

[29] Moustafa, M., Mohd, M.H., Ismail, A.I. \& Abdullah, F.A. (2020). Dynamical analysis of a fractional-order eco-epidemiological model with disease in prey population. Advances in Difference Equations, 2020(1), 1-24. 
[30] Singh, J., et al. (2021) Analysis of fractional model of guava for biological pest control with memory effect. Journal of Advanced Research , 32, 99-108.

[31] Tuan, N.H., Tri, V.V., Singh, J. \& Thach, T.N. (2020). On a fractional Rayleigh-Stokes equation driven by fractional Brownian motion. Mathematical Methods in the Applied Sciences, 2020, 1-16.

[32] Abu Arqub, O., et al. (2021). Reproducing kernel approach for numerical solutions of fuzzy fractional initial value problems under the Mittag-Leffler kernel differential operator. Mathematical Methods in the Applied Sciences, 1-22.

[33] Bonyah, E., et al. (2021). Fractional optimal control dynamics of coronavirus model with Mittag-Leffler law. Ecological Complexity, 45, 100880.

[34] Chattopadhyay, J. \& Bairagi, N. (2001). Pelicans at risk in Salton sea-an ecoepidemiological model. Ecological Modelling, 136(2-3), 103-112.

[35] Chattopadhyay, J., Srinivasu, P.D.N. \& Bairagi, N. (2003). Pelicans at risk in Salton Sea - an eco-epidemiological model-II. Ecological Modelling, 167(1-2), 199-211.

[36] Greenhalgh, D. \& Haque, M. (2007). A predator-prey model with disease in the prey species only. Mathematical Methods in the Applied Sciences, 30(8), 911-929.

[37] Greenhalgh, D., Khan, Q.J. \& Pettigrew, J.S. (2017). An eco-epidemiological predator-prey model where predators distinguish between susceptible and infected prey. Mathematical Methods in the Applied Sciences, 40(1), 146166.

[38] Odibat, Z. \& Shawagfeh, N.(2007) Generalized Taylors formula. Appl. Math. Comput., 186, 286-293

[39] Vargas-De-León, C. (2015). Volterra-type Lyapunov functions for fractional-order epidemic systems. Communications in Nonlinear Science and Numerical Simulation, 24(1-3), 75-85.

[40] Aziz-Alaoui, M.A. (2002). Study of a Leslie-Gower-type tritrophic population model. Chaos, Solitons \& Fractals, 14(8),1275-1293.

[41] Roberts, M.G. \& Heesterbeek, J.A.P. (2013). Characterizing the next-generation matrix and basic reproduction number in ecological epidemiology. Journal of Mathematical Biology, 66(4-5), 1045-1064.

[42] Huo, J., Zhao, H. \& Zhu, L. (2015). The effect of vaccines on backward bifurcation in a fractional-order HIV model. Nonlinear Analysis: Real World Applications, 26, 289-305.

[43] Garrappa, R. (2018) Numerical solution of fractional differential equations: A survey and a software tutorial. Mathematics, 6(2), 16.

[44] Li, X. \& Wu, R. (2014). Hopf bifurcation analysis of a new commensurate fractionalorder hyperchaotic system. Nonlinear Dynamics, 78(1), 279-288.

[45] Das, M. \& Samanta, G.P. (2021). A preypredator fractional order model with fear effect and group defense. International Journal of Dynamics and Control, 9(1), 334-349.

[46] Wu, S.X., \& Meng, X.Y. (2021). Dynamics of a delayed predator-prey system with fear effect, herd behavior and disease in the susceptible prey. AIMS Mathematics, 6(4), 36543685.

[47] Pal, AK. (2020). Effect of fear on a modified Lesli-Gower predator-prey ecoepidemiological model with disease in predator. Journal of Applied Mathematics 83 Informatics, 38(5-6), 375-406.

[48] Mandal, M., et al. (2020). Modelling and control of a fractional-order epidemic model with fear effect. Energy, Ecology and Environment, $5(6), 421-432$.

[49] Sha, A., et al. (2019). Backward bifurcation, oscillations and chaos in an ecoepidemiological model with fear effect. Journal of Biological Dynamics, 13(1), 301-327.

Ankur Jyoti Kashyap is a research scholar in the Department of Mathematics, Gauhati University, Assam, India.

(10) https://orcid.org/0000-0002-7827-3290

Debasish Bhattacharjee is an Assistant Professor in the Department of Mathematics, Gauhati University, Assam, India.

(D) https://orcid.org/0000-0003-0817-9562

Hemanta Kumar Sarmah is a Professor in the Department of Mathematics, Gauhati University, Assam, India.

(10) https://orcid.org/0000-0001-7124-5564 


\section{Appendix A.}

Coefficients of $S^{*}$ in 20

$$
\begin{aligned}
\rho_{0} & =f k m(h+2 m), \\
\rho_{1} & =-c_{1} f k(h+3 m)-f k p_{1}(h+m)-(h+2 m)\left(h r_{1}(h+m)-k m\left(-3 \gamma_{1} f+h+m\right)\right), \\
\rho_{2} & =c_{1}\left(-k\left(-2 \gamma_{1} f(h+3 m)+h^{2}+5 h m+5 m^{2}\right)+f k p_{1}+h r_{1}(2 h+3 m)\right)+c_{1}^{2} f k \\
& -k p_{1}(h+m)\left(-3 \gamma_{1} f+h+m\right)+(h+2 m)\left(r_{1}(h+m)\left(3 \gamma_{1} h+k(h+m)\right)\right. \\
& -3 \gamma_{1} k m\left(-\gamma_{1} f+h+m\right), \\
\rho_{3} & =c_{1}\left(-\left(\gamma_{1} k\left(\gamma_{1} f(h+3 m)-2\left(h^{2}+5 h m+5 m^{2}\right)\right)-2 k p_{1}\left(-\gamma_{1} f+h+m\right)\right.\right. \\
& +r_{1}\left(k\left(3 h^{2}+8 h m+5 m^{2}\right)+2 \gamma_{1} h(2 h+3 m)\right)-c_{1}^{2}\left(k\left(\gamma_{1} f-2(h+2 m)\right)+h r_{1}\right) \\
& -\gamma_{1}\left((h+2 m)\left(\gamma_{1} k m\left(\gamma_{1} f-3(h+m)\right)+3 r_{1}(h+m)\left(\gamma_{1} h+k(h+m)\right)\right)\right. \\
& -3 k p_{1}(h+m)\left(-\gamma_{1} f+h+m\right), \\
\rho_{4} & =-\left(c_{1}+\gamma_{1}(h+m)\right)\left(c_{1}\left(\gamma_{1} k(h+3 m)-r_{1}\left(\gamma_{1} h+3 h k+4 k m\right)+k p_{1}\right)+c_{1}^{2} k\right. \\
& -\gamma_{1}\left(k p_{1}\left(\gamma_{1} f-3(h+m)\right)+(h+2 m)\left(r_{1}\left(\gamma_{1} h+3 k(h+m)\right)-\gamma_{1} k m\right)\right), \\
\rho_{5} & =-k\left(c_{1}+\gamma_{1}(h+m)\right)^{2}\left(c_{1} r_{1}+\gamma_{1}\left(r_{1}(h+2 m)-p_{1}\right)\right) .
\end{aligned}
$$

\section{Proof of Theorem 5}

Proof. At the equilibrium point $E_{3}$ the system (6) reduces to,

$$
\begin{aligned}
r_{1} S_{3}\left(1-\frac{S_{3}+I_{3}}{k}\right)\left(\frac{1}{1+f Y_{3}}\right)-S_{3} I_{3}-\frac{p_{1} Y_{3} S_{3}}{m Y_{3}+S_{3}} & =0 \\
\delta_{1} Y_{3}\left(1-\frac{h Y_{3}}{S_{3}+I_{3}}\right) & =0 .
\end{aligned}
$$

Consider the Lyapunov function,

$$
\mathcal{W}(S, I, Y)=N_{1}\left(S-S_{3}-S_{3} \ln \frac{S}{S_{3}}\right)+N_{2} I+N_{3}\left(Y-Y_{3}-Y_{3} \ln \frac{Y}{Y_{3}}\right) .
$$

We calculate the $\alpha$-order derivative of $\mathcal{W}(S, I, Y)$ along the solution of the system (6) and applying Lemma 5 we get,

$$
\begin{aligned}
& { }_{t_{0}}^{c} D_{t}^{\alpha} \mathcal{W}(S, I, Y) \\
& =N_{1}\left(\frac{S-S_{3}}{S}\right){ }_{t_{0}}^{c} D_{t}^{\alpha} S(t)+N_{2 t_{0}}^{c} D_{t}^{\alpha} I(t)+N_{3}\left(\frac{Y-Y_{3}}{Y}\right){ }_{t_{0}}^{c} D_{t}^{\alpha} Y(t) \\
& =N_{1}\left(\frac{S-S_{3}}{S}\right)\left[r_{1} S\left(1-\frac{S+I}{k}\right)\left(\frac{1}{1+f Y}\right)-S I-\frac{p_{1} Y S}{m Y+S}\right] \\
& +N_{2}\left(S I-\frac{c_{1} Y I}{m Y+I}-\gamma_{1} I\right)+N_{3}\left(\frac{Y-Y_{3}}{Y}\right)\left[\delta_{1} Y\left(1-\frac{h Y}{S+I}\right)\right] \\
& \leq N_{1}\left(S-S_{3}\right)\left[r_{1}\left(1-\frac{S+I}{k}\right)-I-\frac{p_{1} Y}{m Y+S}-r_{1}\left(1-\frac{S_{3}}{k}\right) \frac{1}{1+f Y_{3}}\right. \\
& \left.+\frac{p_{1} Y_{3}}{m Y_{3}+S_{3}}\right]+N_{2}\left(S I-\frac{c_{1} Y I}{m Y+I}-\gamma_{1} I\right)+N_{3}\left(Y-Y_{3}\right)\left[\delta_{1}\left(1-\frac{h Y}{S+I}\right)\right. \\
& -\delta_{1}\left(1-\frac{h Y_{3}}{S_{3}}\right)
\end{aligned}
$$




$$
\begin{aligned}
& \leq N_{1}\left(S-S_{3}\right)\left[\frac{r_{1} f Y_{3}}{k\left(1+f Y_{3}\right)}-\frac{r_{1}(S+I)}{k}+\frac{r_{1} S_{3}}{k\left(1+f Y_{3}\right)}-I-\frac{p_{1} Y}{m Y+S}\right. \\
& \left.+\frac{p_{1} Y_{3}}{m Y_{3}+S_{3}}\right]+N_{2}\left(S I-\frac{c_{1} Y I}{m Y+I}-\gamma_{1} I\right)+N_{3} \delta_{1} h\left(Y-Y_{3}\right)\left[\frac{-\delta_{1} h Y}{S+I}+\frac{\delta_{1} h Y_{3}}{S_{3}+I_{3}}\right] \\
& \leq N_{1}\left(S-S_{3}\right)\left[\frac{r_{1} f Y_{3}}{k\left(1+f Y_{3}\right)}-\left(\frac{r_{1}}{k}\right) \frac{\left(S-S_{3}\right)+I+f Y_{3}(S+I)}{\left(1+f Y_{3}\right)}-I+\frac{p_{1}\left(S-S_{3}\right)}{m^{2} Y_{3}}\right. \\
& \left.-\frac{p_{1} S_{3}\left(S-S_{3}\right)\left(Y-Y_{3}\right)}{(m Y+S)\left(m Y_{3}+S_{3}\right)}\right]+N_{2}\left(S I-\frac{c_{1} Y I}{m Y+I}-\gamma_{1} I\right)+N_{3} \delta_{1} h\left(Y-Y_{3}\right) \\
& {\left[\frac{Y\left(S-S_{3}\right)+Y\left(I-I_{3}\right)}{(S+I)\left(S_{3}+I_{3}\right)}-\frac{\left(Y-Y_{3}\right)}{S_{3}+I_{3}}\right]} \\
& \leq \frac{f N_{1} r_{1}\left(S-S_{3}\right) Y_{3}}{k\left(f Y_{3}+1\right)}-\frac{r_{1}}{k}\left(S-S_{3}\right)^{2}-N_{1}\left(S-S_{3}\right)\left[I+\frac{I+f Y_{3}(S+I)}{1+f Y_{3}}\right] \\
& +\frac{N_{1} p_{1}}{m^{2} Y_{3}}\left(S-S_{3}\right)^{2}-\left(S-S_{3}\right)\left(Y-Y_{3}\right) \frac{N_{1} P_{1} S_{3}}{(m Y+S)\left(m Y_{3}+S_{3}\right)}-\frac{N_{3} \delta_{1} h S\left(Y-Y_{3}\right)^{2}}{(S+I) S_{3}} \\
& +\frac{\left(S-S_{3}\right)\left(Y-Y_{3}\right) N_{3} \delta_{1} h}{(S+I) S_{3}}+\frac{N_{3} \delta_{1} h Y_{3}\left(Y-Y_{3}\right)}{S_{3}}+N_{2}\left(S I-\frac{c_{1} Y I}{m Y+I}-\gamma_{1} I\right) \\
& \leq \frac{N_{1} r_{1} f\left(S-S_{3}\right) Y_{3}}{k\left(f Y_{3}+1\right)}+N_{1}\left(S-S_{3}\right)^{2}\left[\frac{p_{1}}{m^{2} Y_{3}}-\frac{r_{1}}{k}\right]-\frac{N_{3} \delta_{1} h S}{(S+I) S_{3}}\left(Y-Y_{3}\right)^{2} \\
& +\left[\left(S-S_{3}\right)\left(Y-Y_{3}\right)\left\{\frac{N_{3} \delta_{1} h}{(S+I) S_{3}}-\frac{N_{1} p_{1} S}{(m Y+S)\left(m Y_{3}+S_{3}\right)}\right\}\right. \\
& \left.+N_{1} S_{3}\left\{I+\frac{I+f Y_{3}(S+I)}{1+f Y_{3}}\right\}+\frac{N_{3} \delta_{1} h Y_{3} Y}{S_{3}}-N_{1} S\left\{I+\frac{I+f Y_{3}(S+I)}{1+f Y_{3}}\right\}\right] \\
& +N_{2}\left(S I-\frac{c_{1} Y I}{m Y+I}-\gamma_{1} I\right) \cdot
\end{aligned}
$$

Suppose $\frac{p_{1}}{m^{2} Y_{3}}<\frac{r_{1}}{k}$ and $\theta_{1}<S, I, Y<\theta_{2}$. We choose $N_{1}$ and $N_{3}$ such that

$$
\begin{aligned}
& \frac{N_{3}}{N_{1}}>\min \left\{\frac{2 \theta_{2} S_{3}\left(\theta_{2}^{2}(m+1)\left(f Y_{3}+2\right)\left(m Y_{3}+S_{3}\right)+\theta_{2} p_{1} Y_{3}\left(f Y_{3}+1\right)\right)}{\delta_{1} h \theta_{1}^{2}(m+1)\left(f Y_{3}+1\right)\left(m Y_{3}+S_{3}\right)}, \frac{2 \theta_{2}^{3} p_{1} S_{3}^{2}}{\delta_{1} h \theta_{1}^{2}(m+1) Y_{3}}\right\}, \\
& \frac{N_{3}}{N_{1}}<\frac{2 \theta_{2}^{3} S_{3}\left(\theta_{2}-f r_{1} Y_{3}\right)}{\delta_{1} h\left(f Y_{3}+1\right)\left(Y_{3}\left(\theta_{1}^{2}+S_{3}\right)+2 \theta_{1}^{2}\left(Y_{3}+1\right)\right)} .
\end{aligned}
$$

Then ${ }_{t_{0}}^{c} D_{t}^{\alpha} \mathcal{W}(S, I, Y)<N_{2}\left(S I-\frac{c_{1} Y I}{m Y+I}-\gamma_{1} I\right)$.

Clearly, ${ }_{t_{0}}^{C} D_{t}^{\alpha} \mathcal{W}(S, I, Y) \leq 0$ when $S_{3}-\frac{c_{1}}{m}-\gamma_{1}<0$ which is equivalent to $\Re_{0}<k\left(S_{3}-\frac{c_{1}}{m}\right)^{-1}$

Hence the proof.

\section{Proof of Theorem 6;}

Proof. At the equilibrium point $E^{*}$ system (6) reduces to,

$$
\begin{aligned}
r_{1} S^{*}\left(1-\frac{S^{*}+I^{*}}{k}\right)\left(\frac{1}{1+f Y^{*}}\right)-S^{*} I^{*}-\frac{p_{1} Y^{*} S^{*}}{m Y^{*}+S^{*}} & =0, \\
S^{*} I^{*}-\frac{c_{1} Y^{*} I^{*}}{m Y^{*}+I^{*}}-\gamma_{1} I^{*} & =0, \\
\delta_{1} Y^{*}\left(1-\frac{h Y^{*}}{S^{*}+I^{*}}\right) & =0 .
\end{aligned}
$$

To study the globally asymptotically stability of $E^{*}$ the following positive definite Lyapunov function is considered:

$$
\mathcal{W}(S, I, Y)=L_{1}\left(S-S^{*}-S^{*} \ln \frac{S}{S^{*}}\right)+L_{2}\left(I-I^{*}-I^{*} \ln \frac{I}{I^{*}}\right)+L_{3}\left(Y-Y^{*}-Y^{*} \ln \frac{Y}{Y^{*}}\right) .
$$


We calculate the $\alpha$-order derivative of $\mathcal{W}(S, I, Y)$ along the solution of the system (6) and applying Lemma 5 we get,

$$
\begin{aligned}
& { }_{t_{0}}^{c} D_{t}^{\alpha} \mathcal{W}(S, I, Y)=L_{1} \frac{S-S^{*}}{S}{ }_{t_{0}}^{c} D_{t}^{\alpha} S(t)+L_{2} \frac{I-I^{*}}{I}{ }_{t_{0}}^{c} D_{t}^{\alpha} I(t)+L_{3} \frac{Y-Y^{*}}{Y}{ }_{t_{0}}^{c} D_{t}^{\alpha} Y(t) \\
& =L_{1} \frac{S-S^{*}}{S}\left[r_{1} S\left(1-\frac{S+I}{k}\right)\left(\frac{1}{1+f Y}\right)-S I-\frac{p_{1} Y S}{m Y+S}\right] \\
& +L_{2} \frac{I-I^{*}}{I}\left[S I-\frac{c_{1} Y I}{m Y+I}-\gamma_{1} I\right]+L_{3} \frac{Y-Y^{*}}{Y}\left[\delta_{1} Y\left(1-\frac{h Y}{S+I}\right)\right] \\
& \leq L_{1}\left(S-S^{*}\right)\left[r_{1}\left(1-\frac{S+I}{k}\right)-I-\frac{p_{1} Y}{m Y+S}\right. \\
& \left.-r_{1}\left(1-\frac{S^{*}+I^{*}}{k}\right)\left(\frac{1}{1+f Y^{*}}\right)+I^{*}+\frac{p_{1} Y^{*}}{m Y^{*}+S^{*}}\right] \\
& +L_{2}\left(I-I^{*}\right)\left[S-\frac{c_{1} Y}{m Y+I}-\gamma_{1}-S^{*}+\frac{c_{1} Y^{*}}{m Y^{*}+I^{*}}+\gamma_{1}\right] \\
& +L_{3}\left(Y-Y^{*}\right)\left[\delta_{1}\left(1-\frac{h Y}{S+I}\right)-\delta_{1}\left(1-\frac{h Y^{*}}{S^{*}+I^{*}}\right)\right] \\
& \leq \frac{L_{1} r_{1} f Y^{*}}{k\left(1+f Y^{*}\right)}\left(S-S^{*}\right)-\frac{L_{1} r_{1}}{k\left(1+f Y^{*}\right)}\left(S-S^{*}\right)^{2}-L_{1}\left(1+\frac{r_{1}}{k\left(1+f Y^{*}\right)}\right) \\
& \left(S-S^{*}\right)\left(I-I^{*}\right)+\frac{L_{1} p_{1}}{m^{2} Y^{*}}\left(S-S^{*}\right)^{2}-\frac{L_{1} p_{1} S}{(m Y+S)\left(m Y^{*}+S^{*}\right)}\left(Y-Y^{*}\right)\left(S-S^{*}\right) \\
& +L_{2}\left(I-I^{*}\right)\left(S-S^{*}\right)+\frac{L_{2} c_{1}}{m^{2} Y^{*}}\left(I-I^{*}\right)^{2}-\frac{L_{2} c_{1} I\left(Y-Y^{*}\right)\left(I-I^{*}\right)}{(m Y+I)\left(m Y^{*}+I^{*}\right)} \\
& +\delta_{1} h L_{3}\left[\frac{\left(S-S^{*}\right)\left(Y-Y^{*}\right)}{(S+I)\left(S^{*}+I^{*}\right)}-\frac{\left(Y-Y^{*}\right)^{2}(S-I)}{(S+I)\left(S^{*}+I^{*}\right)}-\frac{Y\left(I-I^{*}\right)\left(Y-Y^{*}\right)}{(S+I)\left(S^{*}+I^{*}\right)}\right] \\
& \leq \frac{L_{1} r_{1} f Y^{*}}{k\left(1+f Y^{*}\right)}\left(S-S^{*}\right)-\frac{L_{1} r_{1}}{k\left(1+f Y^{*}\right)}\left(S-S^{*}\right)^{2}-\left(S-S^{*}\right)\left(I-I^{*}\right) \\
& {\left[L_{1}\left(1+\frac{r_{1}}{k\left(1+f Y^{*}\right)}\right)-L_{2}\right]+\frac{L_{1} p_{1}}{m^{2} Y^{*}}\left(S-S^{*}\right)^{2}+\frac{L_{2} c_{1}}{m^{2} Y^{*}}\left(I-I^{*}\right)^{2}} \\
& -\frac{L_{1} p_{1} S}{(m Y+S)\left(m Y^{*}+S^{*}\right)}\left(Y-Y^{*}\right)\left(S-S^{*}\right)-\frac{L_{2} c_{1} I\left(Y-Y^{*}\right)\left(I-I^{*}\right)}{(m Y+I)\left(m Y^{*}+I^{*}\right)} \\
& +\delta_{1} h L_{3}\left[\frac{\left(S-S^{*}\right)\left(Y-Y^{*}\right)}{(S+I)\left(S^{*}+I^{*}\right)}-\frac{\left(Y-Y^{*}\right)^{2}(S-I)}{(S+I)\left(S^{*}+I^{*}\right)}-\frac{Y\left(I-I^{*}\right)\left(Y-Y^{*}\right)}{(S+I)\left(S^{*}+I^{*}\right)}\right] \\
& \leq \frac{L_{1} r_{1} f Y^{*}}{k\left(1+f Y^{*}\right)}\left(S-S^{*}\right)-\left[\frac{L_{1} r_{1}}{k\left(1+f Y^{*}\right)}-\frac{L_{1} p_{1}}{m^{2} Y^{*}}\right]\left(S-S^{*}\right)^{2}-\left(S-S^{*}\right)\left(I-I^{*}\right) \\
& {\left[L_{1}\left(1+\frac{r_{1}}{k\left(1+f Y^{*}\right)}\right)-L_{2}\right]+\frac{L_{2} c_{1}}{m^{2} Y^{*}}\left(I-I^{*}\right)^{2}} \\
& -\frac{L_{1} p_{1} S}{(m Y+S)\left(m Y^{*}+S^{*}\right)}\left(Y-Y^{*}\right)\left(S-S^{*}\right)-\frac{L_{2} c_{1} I\left(Y-Y^{*}\right)\left(I-I^{*}\right)}{(m Y+I)\left(m Y^{*}+I^{*}\right)} \\
& +\delta_{1} h L_{3}\left[\frac{\left(S-S^{*}\right)\left(Y-Y^{*}\right)}{(S+I)\left(S^{*}+I^{*}\right)}-\frac{\left(Y-Y^{*}\right)^{2}(S-I)}{(S+I)\left(S^{*}+I^{*}\right)}-\frac{Y\left(I-I^{*}\right)\left(Y-Y^{*}\right)}{(S+I)\left(S^{*}+I^{*}\right)}\right] \\
& \leq-\left[\frac{L_{1} r_{1}}{k\left(1+f Y^{*}\right)}-\frac{L_{1} p_{1}}{m^{2} Y^{*}}\right]\left(S-S^{*}\right)^{2}-\left(S-S^{*}\right)\left(I-I^{*}\right) \\
& \left(L_{1}\left(1+\frac{r_{1}}{k\left(1+f Y^{*}\right)}\right)-L_{2}\right)
\end{aligned}
$$




$$
\begin{aligned}
& +\left[\frac{L_{1} r_{1} f Y^{*}}{k\left(1+f Y^{*}\right)}\left(S-S^{*}\right)+\frac{L_{2} c_{1}}{m^{2} Y^{*}}\left(I-I^{*}\right)^{2}\right. \\
& -\frac{L_{1} p_{1} S}{(m Y+S)\left(m Y^{*}+S^{*}\right)}\left(Y-Y^{*}\right)\left(S-S^{*}\right)-\frac{L_{2} c_{1} I\left(Y-Y^{*}\right)\left(I-I^{*}\right)}{(m Y+I)\left(m Y^{*}+I^{*}\right)} \\
& \left.+\delta_{1} h L_{3}\left\{\frac{\left(S-S^{*}\right)\left(Y-Y^{*}\right)}{(S+I)\left(S^{*}+I^{*}\right)}-\frac{\left(Y-Y^{*}\right)^{2}(S-I)}{(S+I)\left(S^{*}+I^{*}\right)}-\frac{Y\left(I-I^{*}\right)\left(Y-Y^{*}\right)}{(S+I)\left(S^{*}+I^{*}\right)}\right\}\right] .
\end{aligned}
$$

Suppose

and $\theta_{1}<S, I, Y<\theta_{2}$.

$$
L_{2}=L_{1}\left(1+\frac{r_{1}}{k\left(1+f Y^{*}\right)}\right) \frac{r_{1}}{k\left(1+f Y^{*}\right)}>\frac{p_{1}}{m^{2} Y^{*}}
$$

We choose $L_{1}$ and $L_{3}$ such that

$$
\frac{2 \theta_{2}^{2} \theta_{2} p_{1} S^{*}\left(I^{*}+S^{*}\right)\left(S^{*}+Y^{*}\right)}{\delta_{1} \theta_{1}^{4} h(m+1)\left(m Y^{*}+S^{*}\right)}<\frac{L_{3}}{L_{1}}<\frac{2 \theta_{2} p_{1} S^{* 2} Y^{*}\left(I^{*}+S^{*}\right)}{\delta_{1} h \theta_{1}(m+1)\left(m Y^{*}+S^{*}\right)\left(Y^{*}\left(I Y^{*}+S^{*}\right)+\theta_{1}^{2}\left(2 Y^{*}+1\right)\right)} \text {. }
$$

Then ${ }_{t_{0}}^{c} D_{t}^{\alpha} \mathcal{W}(S, I, Y)<0$.

Expressions of $\omega_{i},(i=1,2,3)$ in equation (24),

$$
\begin{aligned}
& \omega_{1}=\frac{c_{1} m z^{2}}{(m z+y)^{2}}+\gamma_{1}-\frac{r_{1}(k-2 x-y)}{f k z+k}-\frac{\delta_{1}(-2 h z+x+y)}{x+y}+\frac{m p_{1} z^{2}}{(m z+x)^{2}}-x+y, \\
& \omega_{2}=-\frac{c_{1} m r_{1} z^{2}(k-2 x-y)}{(f k z+k)(m z+y)^{2}}+\frac{c_{1} \delta_{1} h y^{2} z^{2}}{(x+y)^{2}(m z+y)^{2}}-\frac{c_{1} \delta_{1} m z^{2}(-2 h z+x+y)}{(x+y)(m z+y)^{2}}+\frac{c_{1} m^{2} p_{1} z^{4}}{(m z+x)^{2}(m z+y)^{2}} \\
& +\frac{c_{1} m y z^{2}}{(m z+y)^{2}}-\frac{\delta_{1} h x z^{2}\left(\frac{f r_{1}(-k+x+y)}{k(f z+1)^{2}}-\frac{p_{1} x}{(m z+x)^{2}}\right)}{(x+y)^{2}}-\frac{\delta_{1} r_{1}(-k+2 x+y)(-2 h z+x+y)}{(x+y)(f k z+k)} \\
& -\frac{\gamma_{1} r_{1}(k-2 x-y)}{f k z+k}+\frac{x y\left(f k z+k+r_{1}\right)}{f k z+k}-\frac{r_{1} x(-k+2 x+y)}{f k z+k}-\frac{\delta_{1} m p_{1} z^{2}(-2 h z+x+y)}{(x+y)(m z+x)^{2}} \\
& -\frac{\gamma_{1} \delta_{1}(-2 h z+x+y)}{x+y}+\frac{\delta_{1} x(-2 h z+x+y)}{x+y}-\frac{\delta_{1} y(-2 h z+x+y)}{x+y}+\frac{\gamma_{1} m p_{1} z^{2}}{(m z+x)^{2}}-\frac{m p_{1} x z^{2}}{(m z+x)^{2}} \\
& -x y+\gamma_{1} y \text {, } \\
& \omega_{3}=\left(-\frac{\delta_{1}}{(x+y)^{2}}\right)\left[\frac{c_{1} m y z^{2}(x+y)(-2 h z+x+y)}{(m z+y)^{2}}-\frac{c_{1} h y^{3} z^{2}}{(m z+y)^{2}}-x y(x+y)(-2 h z+x+y)\right. \\
& +\frac{c_{1} m^{2} p_{1} z^{4}(x+y)(-2 h z+x+y)}{(m z+x)^{2}(m z+y)^{2}}-\frac{c_{1} h m p_{1} y^{2} z^{4}}{(m z+x)^{2}(m z+y)^{2}}-\frac{m p_{1} x z^{2}(x+y)(-2 h z+x+y)}{(m z+x)^{2}} \\
& +\frac{c_{1} h r_{1} y^{2} z^{2}(k-2 x-y)}{(f k z+k)(m z+y)^{2}}+\frac{r_{1} x(x+y)(k-2 x-y)(-2 h z+x+y)}{f k z+k} \\
& +\frac{x y(x+y)\left(f k z+k+r_{1}\right)(-2 h z+x+y)}{f k z+k}-\frac{c_{1} m r_{1} z^{2}(x+y)(k-2 x-y)(-2 h z+x+y)}{(f k z+k)(m z+y)^{2}} \\
& +\frac{c_{1} h x y^{2} z^{2}\left(f k z+k+r_{1}\right)}{(f k z+k)(m z+y)^{2}}-h x y z^{2}\left(\frac{p_{1} x}{(m z+x)^{2}}-\frac{f r_{1}(-k+x+y)}{k(f z+1)^{2}}\right) \\
& +\frac{c_{1} h m x z^{4}\left(\frac{f r_{1}(-k+x+y)}{k(f z+1)^{2}}-\frac{p_{1} x}{(m z+x)^{2}}\right)}{(m z+y)^{2}}-h x^{2} z^{2}\left(\frac{f r_{1}(-k+x+y)}{k(f z+1)^{2}}-\frac{p_{1} x}{(m z+x)^{2}}\right) \\
& +\frac{\gamma_{1} m p_{1} z^{2}(x+y)(-2 h z+x+y)}{(m z+x)^{2}}+\gamma_{1} y(x+y)(-2 h z+x+y) \\
& \left.+\gamma_{1} h x z^{2}\left(\frac{f r_{1}(-k+x+y)}{k(f z+1)^{2}}-\frac{p_{1} x}{(m z+x)^{2}}\right)-\frac{\gamma_{1} r_{1}(x+y)(k-2 x-y)(-2 h z+x+y)}{f k z+k}\right] .
\end{aligned}
$$


An International Journal of Optimization and Control: Theories \& Applications (http://ijocta.balikesir.edu.tr)

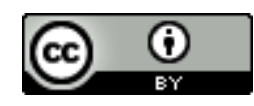

This work is licensed under a Creative Commons Attribution 4.0 International License. The authors retain ownership of the copyright for their article, but they allow anyone to download, reuse, reprint, modify, distribute, and/or copy articles in IJOCTA, so long as the original authors and source are credited. To see the complete license contents, please visit http://creativecommons.org/licenses/by/4.0/. 